\title{
ON SETS OF MATRICES WITH COEFFICIENTS IN A DIVISION RING
}

\author{
BY \\ RICHARD BRAUER
}

A number of recent books deal with the theory of groups of linear transformations and its connection with the theory of algebras $\left({ }^{1}\right)$. Most of the work has been restricted to the case of completely reducible systems or, in other words, to semisimple algebras. There are, however, a number of questions which make it desirable not to neglect the other case. The aim of this and a following paper is a study of such not completely reducible systems, in particular of their regular representations. It appeared necessary to start again right from the beginning of the theory, in order to add a number of remarks to well known results and methods $\left({ }^{2}\right)$. The coefficients of the matrices in this paper are taken from an arbitrary division ring $K$ (=skew field or noncommutative field $K$ ). This is a generalization of the ordinary theory which does not always work smoothly. For instance, the (left) rank of a ring of matrices $\mathfrak{A}$ is not invariant under similarity transformation. This implies that similar rings $\mathfrak{A}$ and $\mathfrak{A}_{1}$ may have different regular representations. Yet it is possible to derive a number of results which, in the case of a commutative $K$, imply the fundamental theorems of Frobenius, Burnside, Loewy, I. Schur and Wedderburn.

Sections 1 and 2 deal with a number of group-theoretical remarks. The first of these are concerned with the Jordan-Hölder theorem. The connection between two composition series is studied more closely, and it is proved that sets of residue systems can be chosen such that they can be used in either composition series. Further, the upper and lower Loewy series of a group are studied. It is shown that the $i$ th factor groups in both have a common constituent. This implies the theorem of Krull and Ore $\left({ }^{3}\right)$ that both series have the same length. In Section 3, the necessary tools from the theory of matrices are described briefly. The following two sections contain an application of the group-theoretical methods to the study of the irreducible and the Loewy constituents of a set of matrices. In Section 6, a number of further remarks are added, for instance a generalization of a theorem of A. H. Clifford $\left.{ }^{4}\right)$.

Presented to the Society, April 16,1938, under the title On groups of linear transformations; received by the editors June 17,1940 .

(1) Cf., for instance, Albert [1, 2], Deuring [7], Murnaghan [17], van der Waerden [28, 29], Wedderburn [30], and, in particular, Weyl [31].

(2) For these results and methods, compare the papers given in the bibliography.

(3) Krull [12] proved this for Abelian groups, Ore [22] in the general case.

(4) Clifford [6]. 
In the second part, the regular representation stands in the foreground and, accordingly, we consider systems $\mathfrak{A}$ of matrices which form semigroups (i.e., are closed under multiplication). There exists a certain reciprocity between $\mathfrak{A}$ and its regular representation $\mathfrak{R}$. In order to show the inner reason for this more clearly, we begin Section 7 with a study of group pairs, first introduced by Pontrjagin $\left({ }^{5}\right)$ in connection with topological investigations. Section 8 deals with the regular representation $\Re$. It is, for instance, shown that $\mathfrak{A}$ and $\Re$ have the same irreducible constituents (except perhaps 0 ); the number of Loewy constituents in both is either the same or differs by one. A number of further results concerning the distribution of the irreducible parts of the Loewy constituents of $\mathfrak{Y}$ ? are proved.

It now follows that the (left) rank $r$ of an irreducible semigroup $\mathfrak{A}$ is divisible by the degree $n$. The quotient $r / n$ can be expressed by means of properties of the commuting set (Section 9). This furnishes the basis for the proof of Wedderburn's theorem, and of the generalized Burnside theorem. In Section 10, representations of sets of matrices as direct sums of subsets are studied. Finally, in Sections 11 and 12, rings $\mathfrak{A}$ of matrices of degree $a$ are considered which contain all the scalar multiples $k I_{a}$ of the unit matrix $I_{a}$ ( $k$ in $K$ ). Here, of course, the structure theory of algebras can be obtained in its full extent. It is proved that if $\mathfrak{B}$ is a representation of degree $b$ of $\mathfrak{A}$ then $\mathfrak{B}$ is a constituent of $a b \times \mathfrak{A}$. We are further interested in the connection between the Loewy decomposition of the regular representation, and the structure of the powers of the radical.

We add here a few remarks concerning the notation: The word ring is used for noncommutative rings. We use the expression " $l$-multiplication" by $a$ (" $r$-multiplication" by $a$ ) in order to express that an element is multiplied on its left side (right side) by $a$. Except in a few places, there would be no restriction in assuming that the system $\mathfrak{A}$ of matrices forms a ring, but it seems more logical to mention only those properties which are actually needed. Thus $\mathfrak{A}$ can first be any system of matrices, later any semigroup (see above), and in the last section it is assumed to be a ring. The zero-matrix, with any number of rows and columns, is denoted by 0 , the unit matrix by $I$, or more clearly by $I_{n}$ if $n$ is the degree. Places in matrices or sets of matrices which are left blank are to be filled out with 0 -matrices, and stars are used for elements in whose form we are not interested.

\section{REMARKS ON COMPOSITION SERIES}

1. We consider groups $(B)$ with a given set of operators $\left(^{6}\right) \Gamma$ which have a finite composition series

$$
B=B_{0} \supset B_{1} \supset \cdots \supset B_{r}=\{1\} .
$$

(5) Pontrjagin [23].

$\left({ }^{6}\right)$ Cf., for instance, van der Waerden [28, vol. 1, §38]. It is easy to extend the definitions 
Let $\mathfrak{S}$ be a second group with the same operators which has a composition series

$$
\mathfrak{S}=\mathfrak{S}_{0} \supset \mathfrak{S}_{1} \supset \cdots \supset \mathfrak{S}_{s}=\{1\} .
$$

We assume that a homomorphism $\theta$ is given which maps $\mathfrak{S}$ upon a normal subgroup $\mathfrak{S}^{*}$ of $\left(\mathfrak{H}, \mathfrak{S}^{*} \subseteq \mathfrak{S}\right.$.

(1.1A) We can choose complete residues systems $\mathfrak{B}_{\rho}$ of $\mathfrak{S}_{\rho-1}\left(\bmod \mathfrak{S}_{\rho}\right)$ and $\mathfrak{Q}_{\sigma}$ of $\mathfrak{S}_{\sigma-1}\left(\bmod \mathfrak{S}_{\sigma}\right),(\rho=1,2, \cdots, r ; \sigma=1,2, \cdots, s)$ such that (a) $\theta$ either maps $\mathfrak{Q}_{\sigma}$ on a $\mathfrak{B}_{\rho}$ in a (1-1) manner and $\mathfrak{H}_{\rho-1} / \mathfrak{H}_{\rho} \simeq \mathfrak{S}_{\sigma-1} / \mathfrak{S}_{\sigma}$, or $\theta$ maps $\mathfrak{Q}_{\sigma}$ on 1. (b) Each $\mathfrak{P}_{\rho}$ is the image of at most one $\mathfrak{Q}_{\sigma}$.

Proof. We denote by $H^{*}$ the image on which $\theta$ maps the element $H$ of $\mathfrak{S}$ Similarly, let $\Omega^{*}$ be the image of an arbitrary subset $\Re$ of $\mathfrak{S}$. We choose arbitrary residue systems $\mathfrak{Q}_{\sigma}$ for $\mathfrak{S}_{\sigma-1}\left(\bmod \mathfrak{S}_{\sigma}\right)$ which contain the unit element. Every $H$ in $\mathfrak{S}$ possesses a unique representation

$$
H=Q_{1} Q_{2} \cdots Q_{s}, \quad Q_{\sigma} \text { in } \mathfrak{\Omega}_{\sigma} ;
$$

we have $\mathfrak{S}_{\sigma}=\mathfrak{Q}_{\sigma+1} \mathfrak{Q}_{\sigma+2} \cdots \mathfrak{Q}_{8}$. If we change $\mathfrak{Q}_{\sigma}$ by multiplying its elements by elements of $\mathfrak{S}_{\sigma}$, we can obtain the most general residue system of $\mathfrak{S}_{\sigma-1}$ $\left(\bmod \mathfrak{S}_{\sigma}\right)$. By a succession of such changes, we shall arrive at a set of residue systems for which (1.1A) holds.

We assume that (1.1A) holds for groups $\$$ which have a shorter composition series than (1). In particular, (1.1A) will be true for $\mathbb{S}_{1}$ in place of $(S)$ If $\mathfrak{S}^{*} \subseteq(S)$, then we may apply $(1.1 \mathrm{~A})$ to $\mathfrak{S}_{1}$ and $\mathfrak{S}$ and see that it also holds for $\left(\mathfrak{S}\right.$ and $\mathfrak{S}$; the residue system $\mathfrak{B}_{1}$ can be taken arbitrarily.

If $\mathfrak{S}^{*}$ is not a subgroup of $\mathfrak{S}_{1}$, then $\mathfrak{S}^{*} \mathfrak{S}_{1}=\mathfrak{S}$. Let $j$ be the first integer for which $\mathfrak{S}_{j}^{*} \mathfrak{S}_{1} \neq\left(\mathfrak{S}\right.$. Then $\mathfrak{S}_{j}^{*} \mathfrak{S}_{1}$ is a proper normal subgroup of $\mathfrak{S}_{j-1}^{*} \mathfrak{S}_{1}=\mathfrak{S}$ which contains $\mathfrak{S}_{1}$. Hence $\mathfrak{S}_{j}^{*} \mathfrak{G}_{1}=\mathfrak{G}_{1}$, i.e., $\mathfrak{S}_{j}{ }^{*} \subseteq \mathfrak{G}_{1}$. We can define a homomorphic mapping of $\mathfrak{S}_{j-1} / \mathfrak{S}_{j}$ upon $\mathfrak{S}_{j-1}^{*} \mathfrak{S}_{1} / \mathfrak{S}_{j}^{*} \mathfrak{G}_{1}=\mathfrak{G}^{\prime} / \mathfrak{G}_{1}$ by

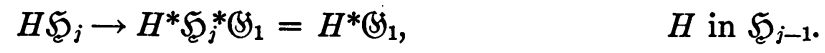

Since $\mathfrak{S}_{j-1} / \mathfrak{S}_{j}$ is simple, this is an isomorphism. It follows that $\mathfrak{Q}_{j}^{*}$ is a complete residue system $\mathfrak{B}_{1}$ of $(S)\left(\bmod \mathfrak{H}_{1}\right)$.

Thus for every $H$ of $\mathfrak{S}$, the element $\left(H^{-1}\right)^{*}$ will lie in some residue class $Q_{j}^{*} \mathfrak{S}_{1}$ with $Q_{j}$ in $\mathfrak{Q}_{j}$, and then $\left(H Q_{j}\right)^{*}$ will lie in $\mathfrak{H}_{1}$. In particular, we can multiply the elements of $\mathfrak{Q}_{\sigma}(\sigma=1,2, \cdots, j-1)$ by such elements of $\mathfrak{Q}_{j}$ that $\theta$ maps the products on elements of $\mathfrak{S}_{1}$. In this manner, we obtain a new residue system of $\mathfrak{S}_{\sigma-1}\left(\bmod \mathfrak{S}_{\sigma}\right)$ which we shall use instead of $\mathfrak{Q}_{\sigma}$ and de-

to the case that the product of an operator $\eta$ with a group element $G$ is defined only if $G$ belongs to a subgroup of $G$ which may depend on $\eta$. When we have a group with operators, we consider only subgroups which are admissible, and homomorphisms and isomorphisms which are operator-homomorphisms and operator-isomorphisms, without always stating this explicitly. We include the case that $\Gamma$ is empty, i.e., that $G$ is a group in the ordinary sense. 
note by $\mathfrak{Q}_{\sigma}$ again. We then have $\mathfrak{Q}_{\sigma}{ }^{*} \subseteq \mathfrak{S}_{1}(\sigma \leqq j-1)$. For $\sigma>j$, we have $\mathfrak{Q}_{\sigma}{ }^{*} \subseteq \mathfrak{S}_{j}{ }^{*} \subseteq \mathfrak{S}_{1}$. Hence $\mathfrak{Q}_{\sigma}{ }^{*} \subseteq \mathfrak{S}_{1}$, for $\sigma \neq j$.

The elements $H$ of $\mathfrak{S}$, whose image $H^{*}$ lies in $\mathfrak{G}_{1}$ form a normal subgroup $\mathfrak{S}^{\prime}$ of $\mathfrak{S}$. Obviously, $\mathfrak{S}^{\prime}$ consists of those elements (3) for which $Q_{j}=1$. If we set $\mathfrak{S C}_{\sigma}^{\prime}=\left[\mathfrak{S}, \mathfrak{S}^{\prime}\right], \mathfrak{S}_{\sigma}^{\prime}$ is obtained from $\mathfrak{S}_{\sigma}=\mathfrak{Q}_{\sigma+1} \mathfrak{Q}_{\sigma+2} \cdots \mathfrak{Q}_{s}$ by removing the factor $\mathfrak{Q}_{j}$ (if it appears). The groups

$$
\mathfrak{S}^{\prime} \supset \mathfrak{S}_{1}^{\prime} \supset \cdots \supset \mathfrak{S}_{j-1}^{\prime}=\mathfrak{S}_{j}^{\prime} \supset \mathfrak{S}_{j+1}^{\prime} \supset \cdots \supset \mathfrak{S}_{s}^{\prime}=\{1\}
$$

form a composition series, and $\mathfrak{Q}_{1}, \cdots, \mathfrak{Q}_{j-1}, \mathfrak{Q}_{j+1}, \cdots, \mathfrak{Q}_{s}$ are a corresponding set of residue systems; $\mathfrak{S}_{\sigma_{-1}}^{\prime} / \mathfrak{S C}_{\sigma}^{\prime} \simeq \mathfrak{S}_{\sigma-1} / \mathfrak{S}_{\sigma}$ for $\sigma \neq j$. Since $\theta$ maps $\mathfrak{S}^{\prime}$ on the normal subgroup $\left[\mathfrak{S}^{*}, \mathfrak{S}_{1}\right]$ of $\mathfrak{S}_{1}$, we may apply the statement $(1.1 \mathrm{~A})$ to the groups $\mathfrak{S}_{1}$ and $\mathfrak{S}^{\prime}$ (in place of $(\mathfrak{S}$ and $\mathfrak{S}$ ), in which case it is assumed to be true. We may have to change the residue classes $\mathfrak{Q}_{1}, \cdots, \mathfrak{Q}_{j-1}, \mathfrak{Q}_{j+1}, \cdots, \mathfrak{Q}_{8}$ still further by multiplying the elements of $\mathfrak{Q}_{\sigma}$ by elements of $\mathfrak{H}_{\sigma}^{\prime}$. But because $\mathfrak{S}_{\sigma}^{\prime} \subseteq \mathfrak{S}_{\sigma}$, this change is also possible in the set of residue classes belonging to (2). This shows that (1.1A) is correct for $\$ 5$ and $\mathfrak{S}\left({ }^{7}\right)$.

At the same time we see

(1.1B) The conditions of (1.1A) can be satisfied by choosing the elements of each $\mathfrak{Q}_{\sigma}$ from a certain subgroup $\mathfrak{S}_{\sigma}$ of $\mathfrak{S}$, and each $\mathfrak{B}_{\rho}$ either as the image of such $a \mathfrak{Q}_{\sigma}$ or as an arbitrary residue system of $\mathfrak{S}_{\rho-1}$ modulo $\mathfrak{S}_{\rho}$.

2. If $\mathfrak{S}^{*}=\mathfrak{H}$, every $\mathfrak{B}_{\rho}$ will appear in the form $\mathfrak{Q}_{\sigma}{ }^{*}$. If, on the other hand, the homomorphism $\theta$ is an isomorphism, every $\mathfrak{Q}_{\sigma}^{*}$ will appear as a $\mathfrak{B}_{\rho}$. We now take $\mathfrak{S}=\mathfrak{S}$ and $\theta$ as the identical mapping. Then (1.1A) gives the JordanHölder theorem and the first part of the following theorem:

(1.2A) If two composition series of $\$$ are given, the residue systems $\mathfrak{B}_{\rho}$ can be chosen such that they can be used in both composition series (in a different arrangement). It is possible to carry one arrangement of the $\mathfrak{P}_{\rho}$ into the other one by successively interchanging two consecutive $\mathfrak{B}_{\rho}$ such that each intermediate arrangement also belongs to a composition series of $B$.

In order to prove the second part, we use the same notation as in $\$ 1.1$. We now have $r=s, \mathfrak{P}_{1}=\mathfrak{Q}_{j}, \mathfrak{S}^{\prime}=\mathfrak{G}_{1}$. The element

$$
Q_{j-1}^{-1} Q_{j}^{-1} Q_{j-1} Q_{j}, \quad Q_{j} \text { in } \mathfrak{Q}_{j}, Q_{j-1} \text { in } \mathfrak{Q}_{j-1},
$$

lies in $\mathfrak{S}^{\prime}$ and in $\mathfrak{S}_{j-1}$, if $j>1$. Since $\left[\mathfrak{S}^{\prime}, \mathfrak{S}_{j-1}\right]=\mathfrak{S}_{j}$, it follows that $Q_{j-1}$ and $Q_{j}$ commute $\left(\bmod \mathfrak{S}_{j}\right)$. If we interchange $\mathfrak{Q}_{j-1}$ and $\mathfrak{Q}_{j}$ in $\mathfrak{Q}_{1}, \mathfrak{Q}_{2}, \cdots, \mathfrak{Q}_{s}$, we obtain a set of residue systems belonging to the composition series

(7) In a similar manner, we can prove a theorem which has the same relation to Schreier's extension of the Jordan-Hölder theorem (Schreier [25], Zassenhaus [32]) as (1.1A) has to the Jordan-Hölder theorem itself. 


$$
\mathfrak{S}=\mathfrak{S}_{0} \supset \mathfrak{S}_{1} \supset \cdots \supset \mathfrak{S}_{j-2} \supset \mathfrak{S}_{j-2}^{\prime} \supset \mathfrak{S}_{j} \supset \cdots \supset \mathfrak{S}_{8}=\{1\}
$$

because $\mathfrak{Q}_{j-1} \mathfrak{Q}_{j} \mathfrak{S}_{j}=\mathfrak{Q}_{j} \mathfrak{Q}_{j-1} \mathfrak{S}_{j}$ and $\mathfrak{H}_{j-2}^{\prime}=\mathfrak{Q}_{j-1} \mathfrak{Q}_{j+1} \mathfrak{Q}_{j+2} \ldots \mathfrak{Q}_{8}$. We next interchange $\mathfrak{Q}_{j}$ with $\mathfrak{Q}_{j-2}$, etc., until $\mathfrak{Q}_{j}$ finally stands at the first place. If $(1.2 \mathrm{~A})$ is true for $\mathfrak{B}_{1}$, as we may assume, it now follows for $\mathfrak{H}^{5}$.

\section{LOEWY SERIES}

1. A group (S) is completely reducible $\left.{ }^{8}\right)$, if it is the direct product of simple groups $\mathfrak{P}_{1}, \mathfrak{P}_{2}, \cdots, \mathfrak{B}_{r}$. As indicated by this notation,

$$
\mathfrak{B}=\mathfrak{B}_{1} \mathfrak{B}_{2} \cdots \mathfrak{B}_{r}, \quad \mathfrak{S}_{1}=\mathfrak{B}_{2} \cdots \mathfrak{B}_{r}, \cdots, \mathfrak{S}_{r-1}=\mathfrak{B}_{r}, \quad \mathfrak{S}_{r}=\{1\}
$$

is a composition series. Every normal simple subgroup $\mathfrak{M}$ of $\mathbb{B}$ is completely reducible and is a direct factor, i.e., $\mathbb{B}=\mathfrak{M} \times \mathfrak{N}$, where $\mathfrak{N}$ is a normal subgroup of $\mathfrak{B}$. Because $\mathfrak{H} / \mathfrak{M} \simeq \mathfrak{N}$, the factor group $\mathbb{S} / \mathfrak{M}$ is also completely reducible.

If $\mathfrak{A}$ is a normal subgroup of an arbitrary group $(\mathfrak{S}$, we say that $\mathfrak{A}$ is completely reducible with regard to $\mathfrak{H}$, if $\mathfrak{A}$ is the direct product of minimal normal subgroups of $\mathfrak{B}$. More generally, if $\mathfrak{A}$ and $\mathfrak{B}$ are normal subgroups of $\mathfrak{B}$ and $\mathfrak{A} \supseteq \mathfrak{B}$, we say that $\mathfrak{A} / \mathfrak{B}$ is completely reducible with regard to $\mathfrak{G}$, if $\mathfrak{A} / \mathfrak{B}$ is completely reducible with regard to $\mathbb{S} / \mathfrak{B}$. If we add the inner automorphism of $(S)$ to the operators of the groups considered (subgroups of $\mathbb{B}$ and factor groups formed out of them), then complete reducibility of $\mathfrak{A} / \mathfrak{B}$ with regard to $(S)$ means the same as ordinary complete reducibility of $\mathfrak{A} / \mathfrak{B}$. In the case of abelian groups (\$), the words "with regard to (S)" can always be omitted.

For any group (\$), we prove easily:

(2.1A) If $\mathfrak{R}$ and $\mathfrak{M}$ are normal subgroups of $(5)$ which are completely reducible with regard to $\mathrm{H}$, the same is true for $\mathfrak{R M}$.

Proof. We add the set of all inner automorphisms of $B$ to the set of operators. If $\mathfrak{D}=[\mathfrak{R}, \mathfrak{M}]$, we may set $\mathfrak{R}=\mathfrak{R}_{1} \times \mathfrak{D}, \mathfrak{M}=\mathfrak{M}_{1} \times \mathfrak{D}$, where $\mathfrak{R}_{1}$ and $\mathfrak{M}_{1}$ are normal subgroups of $\left(\mathfrak{s}\right.$. We then have $\mathfrak{R M}=\mathfrak{R}_{1} \times \mathfrak{M}_{1} \times \mathfrak{D}$, since $\left[\mathfrak{R}_{1}, \mathfrak{M}_{1} \times \mathfrak{D}\right]$ $=\{1\}$. This shows that $(2.1 \mathrm{~A})$ is true.

(2.1B) If $\mathfrak{A}, \mathfrak{B}$ and $\mathfrak{\Im}$ are normal subgroups of $\mathfrak{H}$, where $\mathfrak{B} \subseteq \mathfrak{A}$, and $\mathfrak{A} / \mathfrak{B}$ is completely reducible with regard to $\mathfrak{S}$, then $[\mathfrak{S}, \mathfrak{A}] /[\mathfrak{S}, \mathfrak{B}]$ is completely reducible with regard to $\mathfrak{S}$, and isomorphic with a normal subgroup of $\mathfrak{A} / \mathfrak{B}$.

Proof. We extend the domain of operators as in the proof of (2.1A). The statement is a consequence from the fact that $[\mathfrak{\Im}, \mathfrak{A}] \mathfrak{B} / \mathfrak{B} \simeq[\mathfrak{Y}, \mathfrak{A}] /[\mathfrak{Y}, \mathfrak{A}, \mathfrak{B}]$ $=[\mathfrak{Y}, \mathfrak{A}] /[\mathfrak{\Im}, \mathfrak{B}]$, since $[\mathfrak{\Im}, \mathfrak{A}] \mathfrak{B} / \mathfrak{B}$ is a normal subgroup of $\mathfrak{A} / \mathfrak{B}$.

(2.1C) If $\mathfrak{B}$ and $\mathbb{S}$ are normal subgroups of $\mathfrak{B}$, where $\mathfrak{B C} / \mathfrak{B}$ and $\mathfrak{B C} / \mathfrak{C}$ are both completely reducible with regard to $\mathfrak{B}$, so is $\mathfrak{B C} /[\mathfrak{B}, \mathbb{E}]$.

Proof. From $(2.1 \mathrm{~B})$ it follows that $[\mathfrak{B} \mathfrak{C}, \mathfrak{E}] /[\mathfrak{B}, \mathfrak{E}]=\mathfrak{E} /[\mathfrak{B}, \mathfrak{S}]$ is com-

${ }^{(8)}$ Cf. van der Waerden [28, vol. 1, p. 143]. 
pletely reducible with regard to $\mathfrak{B}$. The same is true for $\mathfrak{B} /[\mathfrak{B}$, $\mathfrak{C}]$. Then (2.1A) shows that $\mathfrak{B C} /[\mathfrak{B}, \mathfrak{C}]$ is completely reducible with regard to $\mathfrak{S} /[\mathfrak{B}, \mathfrak{E}]$, and hence with regard to $(S)$.

2. A Loewy series of $(B)$ is a series of normal subgroups of $(B)$ :

$$
\mathbb{H}=\mathfrak{M}_{0} \supset \mathfrak{M}_{1} \supset \mathfrak{M}_{2} \supset \ldots \supset \mathfrak{M}_{t-1} \supset \mathfrak{M}_{t}=\{1\}
$$

in which each factor group $\mathfrak{M}_{\tau-1} / \mathfrak{M}_{\tau}$ is completely reducible with regard to $\mathfrak{S}$.

Of special importance is the lower Loewy series (or lower cover series of (5)). Here $\mathfrak{M}_{t-1}$ is the normal cover ("Sockel") $\left({ }^{9}\right)$ of $(\$)$, i.e., the union of all minimal normal subgroups of $\mathfrak{B}$. It follows from (2.1A) that $\mathfrak{M}_{t-1}$ is completely reducible with regard to $\mathfrak{S}$. More generally, we take for $\mathfrak{M}_{\tau-1}$ the group for which $\mathfrak{M}_{\tau-1} / \mathfrak{M}_{\tau}$ is the normal cover of $\mathfrak{S} / \mathfrak{M}_{\tau}(\tau=t, t-1, \cdots)$. Then we actually obtain a Loewy series of $\mathfrak{G}$. Obviously, $\mathfrak{M}_{\tau-1}$ is the largest group which can precede $\mathfrak{M}_{\tau}$ in any Loewy series of $(\mathbb{S}$.

Let $\mathfrak{S}$ be a second group, and

$$
\mathfrak{S}=\mathfrak{N}_{0} \supset \mathfrak{R}_{1} \supset \mathfrak{R}_{2} \supset \cdots \supset \mathfrak{N}_{u-1} \supset \mathfrak{N}_{u}=\{1\}
$$

be a Loewy series of $\mathfrak{S}$. We then state

(2.2A) Let $\theta$ be a homomorphic mapping of $\mathfrak{S}$ upon a subgroup $\mathfrak{S}^{*}$ of $(5)$ $\left(\mathfrak{S}^{*} \subseteq \mathfrak{S}\right)$ which maps normal subgroups $\mathfrak{\Re}$ upon normal subgroups $\mathfrak{N}^{*}$ of $\mathfrak{S}^{(10}\left({ }^{10}\right)$. If (5) is the lower Loewy series of $\mathfrak{B}$, and (6) any Loewy series of $\mathfrak{S}$, then

$$
\mathfrak{N}_{u-1}^{*} \subseteq \mathfrak{M}_{t-1}, \mathfrak{N}_{u-2}^{*} \subseteq \mathfrak{M}_{t-2}, \cdots, \mathfrak{N}_{u-\rho}^{*} \subseteq \mathfrak{M}_{t-\rho}, \cdots
$$

Proof. Let $\mathfrak{N}$ be a minimal normal subgroup of $\mathfrak{S}$. If its image $\mathfrak{N}^{*}$ contains a normal subgroup $\mathfrak{T}$ of $\mathfrak{H}$ with $\{1\} \subset \mathfrak{T} \subset \mathfrak{N}^{*}$, the elements of $\mathfrak{N}$ which are mapped upon elements of $\mathfrak{T}$ form a proper subgroup of $\mathfrak{N}$ which is normal in $\mathfrak{S}$. This is impossible, and hence $\mathfrak{N}^{*}$ is a minimal normal subgroup of $\mathfrak{H}$, and belongs therefore to $\mathfrak{M}_{t-1}$, the normal cover of $\mathfrak{B}$. It now follows easily that $\mathfrak{N}_{u-1}^{*} \subseteq \mathfrak{M}_{t-1}$. The mapping $\theta$ induces a homomorphic mapping of $\mathfrak{S} / \mathfrak{N}_{u-1}$ upon a subgroup of $\mathfrak{S} / \mathfrak{M}_{t-1}$, which maps normal subgroups upon normal subgroups. Using the same argument, we obtain $\left(\mathfrak{N}_{u-2} / \mathfrak{N}_{u-1}\right)^{*} \subseteq\left(\mathfrak{M}_{t-2} / \mathfrak{M}_{t-1}\right)$, and hence $\mathfrak{N}_{u \rightarrow 2}^{*} \subseteq \mathfrak{M}_{t-2}$, etc.

3. The dual of the lower Loewy series is the upper Loewy series or upper cover series. Here, $\mathfrak{M}_{\tau}$ is the upper cover of $\mathfrak{M}_{\tau-1}\left({ }^{11}\right)$, i.e., the intersection of all maximal normal subgroups of $\mathfrak{M}_{\tau-1}, \tau=1,2, \cdots$. We see successively that $\mathfrak{M}_{1}, \mathfrak{M}_{2}, \cdots$ are normal in $\mathfrak{S}$. Then $\mathfrak{M}_{\tau}$ can also be defined as the intersection of the normal subgroups of $\mathfrak{B S}$ which are maximal in $\mathfrak{M}_{\tau-1}$. From

(9) Remak [24], Cf. also Ore [22].

${ }^{(10)}$ This assumption is necessary whereas in the dual theorem $(2.3 \mathrm{~A})$ it is sufficient to assume that $\mathfrak{W}^{*}$ is normal in $\mathfrak{S}$.

(11) Ore [22]. 
(2.1C) it follows easily that $\mathfrak{M}_{\tau-1} \angle \mathfrak{M}_{\tau}$ is completely reducible with regard to $\mathfrak{B}$, so that we actually have a Loewy series. Obviously, $\mathfrak{M}_{\tau}$ is the smallest group which can follow $\mathfrak{M}_{\tau-1}$ in any Loewy series of $\mathfrak{B}$.

We now show

(2.3A) Let $\theta$ be a homomorphic mapping of $\mathfrak{S}$ upon a normal subgroup $\mathfrak{S}^{*}$

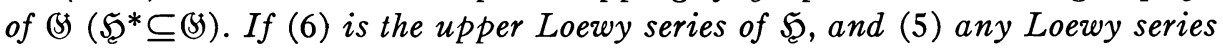
of $\mathfrak{B}$, then $\mathfrak{N}_{\rho}^{*} \subseteq \mathfrak{M}_{\rho}(\rho=1,2, \cdots)$ where $\mathfrak{N}_{\rho}^{*}$ again denotes the image of $\mathfrak{N}_{\rho}$.

Proof. Without restriction, we may assume that to every inner automorphism of $\mathfrak{S}$ there corresponds an operator in $\Gamma$ which produces this automorphism. Form

$$
\overline{\mathbb{S}}=\left[\mathfrak{S}^{*}, \mathfrak{H}\right]=\mathfrak{S}^{*} \supseteq\left[\mathfrak{S}^{*}, \mathfrak{M}_{1}\right] \supseteq\left[\mathfrak{S}^{*}, \mathfrak{M}_{2}\right] \supseteq \cdots \cdot
$$

The distinct groups in $\left(5^{\prime}\right)$ form a Loewy series as follows from (2.1B), and $\theta$ maps $\mathfrak{S}$ upon $\overline{\mathbb{S}}$. We replace $(\mathbb{S}$ by $\overline{(S)}$, and $(5)$ by this Loewy series. If we can prove $(2.3 \mathrm{~A})$ in this case, it also will be true in the original case. It is, therefore, sufficient to prove $(2.3 \mathrm{~A})$ in the case where $\mathfrak{S}=\mathfrak{S}^{*}$. Here, $\mathfrak{N}_{\rho}^{*}$ is a normal subgroup of $\left(\mathfrak{S}\right.$. The totality of elements of $\mathfrak{S}$ whose images lie in $\mathfrak{M}_{1}$ form a normal subgroup $\mathfrak{T}$ of $\mathfrak{S}$. We map $\mathfrak{S} / \mathfrak{T}$ upon $\mathfrak{S} / \mathfrak{M}_{1}$ by $H \mathfrak{T} \rightarrow H^{*} \mathfrak{M}_{1}(H$ in $\mathfrak{S})$. Since $H^{*} \mathfrak{M}_{1}=\mathfrak{M}_{1}$ only if $H$ is in $\mathfrak{T}$, this mapping is an isomorphism. With $\mathfrak{S} / \mathfrak{M}_{1}$, then $\mathfrak{S} / \mathfrak{T}$ also is completely reducible, and hence $\mathfrak{T}$ contains the upper cover $\mathfrak{N}_{1}$ of $\mathfrak{S}$. This implies $\mathfrak{R}_{1}^{*} \subseteq \mathfrak{M}_{1}$. If for $\mathfrak{M}_{1}, \mathfrak{R}_{1}$, and the mapping induced by $\theta$ the statement has been proved, as we may assume, it now follows for $\$$, $\mathfrak{S}$ and the mapping $\theta$.

4. We now consider the case that $\mathfrak{B}=\mathfrak{S}$, and $\theta$ is the identical isomorphism. From (2.2A) it follows that any Loewy series (6) of (5) has at least the same length as the lower Loewy series (5), since for $u<t$ we would have

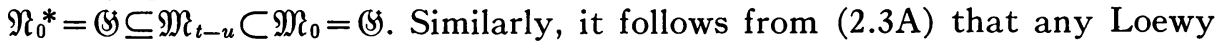
series has at least the same length as the upper Loewy series. (If we use the notation of (5) and (6) for these series, and if we have $t<u$, then $\{1\}=\mathfrak{M}_{t} \supseteq \mathfrak{N}_{t}^{*} \neq\{1\}$ which is impossible.) If we take for (5) the lower and for (6) the upper Loewy series of $\mathbb{S}=\mathfrak{S}$, we have $t=u$, hence

(2.4A) The lower and the upper Loewy series of (5) have the same length ${ }^{12}$ ).

From (2.3A), we obtain $\mathfrak{R}_{\rho} \subseteq \mathfrak{M}_{\rho}$ in our case. If we had $\mathfrak{N}_{\rho-1} \subseteq \mathfrak{M}_{\rho}$, we could apply (2.3A) to the Loewy series

$$
\mathfrak{M}_{\rho} \supset \mathfrak{M}_{\rho+1} \supset \cdots \supset \mathfrak{M}_{u}=\{1\}, \mathfrak{R}_{\rho-1} \supset \mathfrak{N}_{\rho} \supset \cdots \supset \mathfrak{N}_{u}=\{1\},
$$

of which the second one is the upper Loewy series of $\mathfrak{N}_{\rho-1}$. We then find $\mathfrak{N}_{\rho} \subseteq \mathfrak{M}_{\rho+1}, \cdots, \mathfrak{N}_{u-1} \subseteq \mathfrak{M}_{u}=\{1\}$ which is impossible. Consequently, $\mathfrak{N}_{\rho-1}$ contains elements which do not belong to $\mathfrak{M}_{\rho}$, and hence $\mathfrak{N}_{\rho} \subseteq\left[\mathfrak{N}_{\rho-1}, \mathfrak{M}_{\rho}\right] \subset \mathfrak{N}_{\rho-1}$ and $\mathfrak{M}_{\rho} \subset \mathfrak{N}_{\rho-1} \mathfrak{M}_{\rho} \subseteq \mathfrak{M}_{\rho-1}$. Since $\mathfrak{N}_{\rho-1} \mathfrak{M}_{\rho} / \mathfrak{M}_{\rho} \simeq \mathfrak{N}_{\rho-1} /\left[\mathfrak{N}_{\rho-1}, \mathfrak{M}_{\rho}\right]$, we obtain

(2.4B) The pth factor groups $\mathfrak{M}_{\rho-1} / \mathfrak{M}_{\rho}$ and $\mathfrak{N}_{\rho-1} / \mathfrak{N}_{\rho}$ of the lower and upper

${ }^{(12)}$ Krull [12], Ore [22], cf.( ${ }^{(3)}$. 
Loewy series of $(5)$ contain at least one pair of isomorphic normal subgroups $(\neq\{1\})$.

5. We assume now that $B$ is abelian, or, more generally, that all the inner automorphisms of $\$$ belong to the set of operators. We consider a composition series of (\$),

$$
\left(\mathfrak{S}=\mathfrak{S}_{0} \supset \mathfrak{S}_{1} \supset \cdots \supset \mathfrak{S}_{r}=\{1\},\right.
$$

and a corresponding set of residue systems $\mathfrak{B}_{1}, \mathfrak{P}_{2}, \cdots, \mathfrak{P}_{r}$. With regard to a later application, it is desirable to give a method of obtaining the lower Loewy series. It may happen that a $\mathfrak{P}_{\rho}$ can be chosen to be a (normal $\left({ }^{13}\right)$ ) subgroup of $(B)$; we call these $\mathfrak{B}_{\rho}$ the residue systems of lowest kind. We state

(2.5A) The normal cover of $\$ S$ is equal to the product of the residue systems $\mathfrak{B}_{p}$ of lowest kind, if these are chosen to be normal subgroups of $\mathrm{B}$.

Proof. It is clear that all these $\mathfrak{B}_{\rho}$ belong to the normal cover $\mathfrak{S}$ of $\mathfrak{S}$. We determine a set of residue systems $\mathfrak{Q}_{1}, \mathfrak{Q}_{2}, \cdots, \mathfrak{Q}_{8}$ of a composition series of $\mathfrak{S}$ such that each $\mathfrak{Q}_{\sigma}$ is a minimal normal subgroup of (S) (cf. $\$ 2.1$ ), and apply the method of $\$ 1.1$ to $\mathfrak{G}, \mathfrak{S}$, and the identical mapping. If $j$ has the same significance as in $\S 1.1$, we may assume that $j=1$, since the $\mathfrak{Q}_{\sigma}$ here can be permuted arbitrarily. No modification of the $\mathfrak{Q}_{\sigma}$ is necessary, and one $\mathfrak{B}_{\rho}$ can be replaced by $\mathfrak{Q}_{1}$. This shows that this $\mathfrak{B}_{\rho}$ is of lowest kind. After the next step, one $\mathfrak{P}_{\sigma}$ will be replaced by $\mathfrak{Q}_{2}$, etc. Since $\theta$ is a (1-1) mapping, every $\mathfrak{Q}_{\lambda}$ will finally appear. This shows that the number of residue classes of lowest kind cannot be smaller than $s$. The product of these $\mathfrak{B}_{\nu}$, chosen as normal subgroups of $(B)$, must give the full normal cover $\mathfrak{M}_{t-1}$ as stated in (2.5A).

We now remove these $\mathfrak{B}_{\text {v }}$ of lowest kind from $\mathfrak{P}_{1}, \mathfrak{P}_{2}, \cdots, \mathfrak{P}_{r}$ and work from now on modulo $\mathfrak{M}_{t-1}$. It is clear that the remaining $\mathfrak{B}_{\lambda}$ form a system

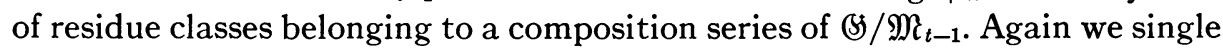
out the residue systems which now are of lowest kind, and choose them such that their elements (mod $\mathfrak{M}_{t-1}$ ) form normal subgroups of $\mathfrak{H} / \mathfrak{M}_{t-1}$. Their product, multiplied by $\mathfrak{M}_{t-1}$ gives the group $\mathfrak{M}_{t-2}$ in the lower Loewy series. Continuing in this manner, we can obtain this series.

\section{Matrices in A Division RING}

1. There is no difficulty in extending the ordinary theory of matrices to the case in which the coefficients of the matrices are taken from a fixed division ring $K$ (instead of a field). Of course, the products $\rho A$ and $A \rho$ of a matrix $A$ and a "scalar" $\rho$ from $K$ will in general be different. Otherwise, there is no difference, as we are not interested in the question of the determinant here. A square matrix $M$ of degree $n$ is nonsingular if there exists a reciprocal $M^{-1}$ with $M M^{-1}=M^{-1} M=I_{n}$ where $I_{n}=\left(\delta_{\kappa \lambda}\right), \delta_{\kappa \kappa}=1, \delta_{\kappa \lambda}=0$ for $\kappa \neq \lambda$, is the unit matrix of degree $n$.

(13) Any admissible subgroup now is normal. 
Let $M_{1}, M_{2}, \cdots, M_{q}$ be matrices of the same type $(m, n)$, i.e., with $m$ rows and $n$ columns. We say that the matrices are $l$-independent, if no linear relation $\alpha_{1} M_{1}+\cdots+\alpha_{q} M_{q}=0$ exists with coefficients $\alpha_{\kappa}$ in $K$, except for $\alpha_{1}=\alpha_{2}=\cdots=\alpha_{q}=0$. Similarly, the matrices are $r$-independent, if no relation $M_{1} \alpha_{1}+\cdots+M_{q} \alpha_{q}=0$ exists, except for $\alpha_{1}=\cdots=\alpha_{q}=0$. The l-rank of a set $\mathfrak{M}$ of matrices of the same type is defined as the maximum number $z$ of $l$-independent matrices of $\mathfrak{M}$, and any $z$ such $l$-independent matrices form an $l$-basis of $\mathfrak{M}$. Correspondingly, the $r$-rank of $\mathfrak{M}$ and $r$-basis of $\mathfrak{M}$ are defined.

2. There is also no difficulty in introducing $n$-dimensional vector-spaces $\mathfrak{B}$ over a division ring $K$, and extending the elementary properties of ordinary vector spaces. We arrange the $n$ components $x_{\kappa}$ of a vector $X$ with regard to a fixed basis in a column (matrix of type $(n, 1)$ ). We consider two operations for vectors, addition and $r$-multiplication with elements of $K$; these operations appear as a special case of the corresponding operations with matrices. The vector space $\mathfrak{B}$ is an abelian group with addition as group-combination, which possesses the elements of $K$ as operators. It is the direct sum of $n$ simple groups.

We may also consider a second set of vectors $U$ which are given by rows (i.e., matrices of type $(1, n)$ ). Here we have an addition and an $l$-multiplication of vectors with elements of $K$. We denote such vectors as contragredient vectors.

A matrix $A=\left(a_{\kappa \lambda}\right)$ of type $(m, n)$ defines a homomorphic mapping of an $n$-dimensional vector space upon a subspace of an $m$-dimensional vector space: $X \rightarrow X^{*}=A X$, provided that in both spaces coordinate systems have been chosen. The matrix $A$ also defines a homomorphic mapping of an $m$ dimensional contragredient space upon a subspace of an $n$-dimensional contragredient space: $U \rightarrow U^{*}=U A$.

3. Let $m=m_{1}+m_{2}+\cdots+m_{k}$ and $n=n_{1}+n_{2}+\cdots+n_{l}$ be partitions of $m$ and $n$. We of ten write matrices $A$ of type $(m, n)$ in the form $\left(A_{\kappa \lambda}\right)$ where $A_{\kappa \lambda}$ itself is a matrix of type $\left(m_{\kappa}, n_{\lambda}\right)$. We then say that $A$ has been broken up according to the scheme $\left(m_{1}, \cdots, m_{k} \mid n_{1}, \cdots, n_{l}\right)$. If $B=\left(B_{\kappa \lambda}\right)$ is a matrix of type $(n, r)$ which is broken up according to a scheme $\left(n_{1}, \cdots, n_{l} \mid r_{1}, \cdots, r_{q}\right)$, then $A B=\left(\sum_{\mu} A_{\kappa \mu} B_{\mu \lambda}\right)$, i.e., the product can be formed as if $A_{\kappa \lambda}$ and $B_{\kappa \lambda}$ are scalars, provided that the right-hand side has a meaning. The corresponding fact holds for sums of matrices; here $A$ and $B$ must be broken up according to the same scheme.

We also break up the $n$-dimensional vector $X$ into an $n_{1}$-dimensional vector $X_{1}$, an $n_{2}$-dimensional vector $X_{2}, \cdots$, an $n_{l}$-dimensional vector $X_{l}$. The matrices of the following linear transformations are of importance.

$$
\begin{aligned}
& T_{i j}(Q): \quad X_{\kappa}^{*}=X_{\kappa} \quad(\kappa \neq i), \quad X_{i}^{*}=X_{i}+Q X_{j} ; \\
& Z_{i j}: \quad X_{\kappa}^{*}=X_{\kappa} \quad(\kappa \neq i, j), \quad X_{i}^{*}=X_{j}, \quad X_{j}^{*}=X_{i} ; \\
& W_{i}(P): \quad X_{\kappa}^{*}=X_{\kappa} \quad(\kappa \neq i), \quad X_{i}^{*}=P X_{i} ;
\end{aligned}
$$


where $Q$ is a matrix of type $\left(n_{i}, n_{j}\right)$, and $P$ a nonsingular matrix of degree $n_{i}$. We denote by $A_{c}$ a matrix in which the columns are broken up according to the scheme $\left(n_{1}, n_{2}, \cdots, n_{l}\right)$, by $A_{r}$ a matrix in which the rows have been broken up in this manner, by $A$ a square matrix in which both rows and columns have been broken up in this manner. By combining the corresponding linear transformations, we obtain easily

(3.3A) The matrix $A_{c} T_{i j}(Q)$ is obtained from $A_{c}$ by adding the ith column, $r$-multiplied by $Q$, to the jth column; $T_{i j}(Q)^{-1} A_{r}$ is obtained from $A_{r}$ by subtracting the jth row, l-multiplied by $Q$, from the ith row. Finally, $T_{i j}(Q)^{-1} A T_{i j}(Q)$ is obtained from $A$ by performing these two operations successively.

(3.3B) The matrix $A_{c} Z_{i j}$ is obtained from $A_{c}$ by interchanging the ith and $j$ th column; $Z_{i j}^{-1} A_{r}$ is obtained from $A_{r}$ by interchanging the ith and $j$ th row; $Z_{i j}^{-1} A Z_{i j}$ is obtained from $A$ by performing both operations.

(3.3C) The matrix $A_{c} W_{i}(P)$ is obtained from $A_{c}$ by $r$-multiplying the ith column by $P ; W_{i}(P)^{-1} A_{r}$ is obtained from $A_{r}$ by l-multiplying the ith row by $P^{-1}$; and $W_{i}(P)^{-1} A W_{i}(P)$ is obtained from $A$ by performing both operations.

4. The operations in $\$ 3.3$ can be used in particular if all the numbers $n_{\lambda}$ are equal to 1 , i.e., if the matrices $A=\left(a_{\kappa \lambda}\right)$ are taken in their original form. We perform with $A$ a succession of operations of the kind mentioned in (3.3A), (3.3B), (3.3C). This amounts to a succession of $l$-multiplications and $r$-multiplications of $A$ by nonsingular square matrices. The new matrix then has the form $G A H$ where $G$ and $H$ are themselves nonsingular square matrices. It can easily be seen that the operations may be chosen such that the new matrix has the form $\left({ }^{14}\right)$

$$
G A H=\left(\begin{array}{ll}
I_{\rho} & 0 \\
0 & 0
\end{array}\right)
$$

Here, $\rho$ is an integer, the rank of $A$; and $\rho \leqq m, \rho \leqq n$.

We now can discuss the solution of linear equations

$$
\sum_{\lambda=1}^{n} a_{\kappa \lambda} x_{\lambda}=b_{\kappa}, \quad \kappa=1,2, \cdots, m,
$$

or, in matrix form, $A X=B$, where $B$ is an $m$-dimensional vector. We set $X=H X^{*}, X^{*}=H^{-1} X$. Then (8) becomes identical with $(G A H) X^{*}=G B$, in which form it can easily be solved because of (7). In particular, in the homogeneous case $B=0$, we have exactly $n-\rho r$-independent solutions $X$ of (8). This shows that the rank $\rho$ of $A$ is uniquely determined by $A$. We may also characterize $\rho$ as the $r$-rank of the set of vectors $B$ which are obtained from

(14) The second row or the second column on the right side may be missing. 
(8), if $X$ ranges over all $n$-dimensional vectors. If the division ring $K$ is replaced by a larger division ring $\bar{K}$, the number $\rho$ remains unchanged, and a complete system of $r$-independent solutions of the homogeneous equations with regard to $K$ will have the corresponding properties with regard to $\bar{K}$. If (8) has no solution in $K$, it has no solution in $\bar{K}$.

The "contragredient" equations

$$
\sum_{k=1}^{m} u_{k} a_{k \lambda}=b_{\lambda}{ }^{\prime}, \quad \lambda=1,2, \cdots, n,
$$

for $u_{1}, u_{2}, \cdots, u_{m}$ can be discussed in a similar manner.

From the characterizations of the rank of a matrix, it follows easily that the rank of a product of matrices is not larger than the rank of either factor.

5. Let us define the transpose $A^{\prime}$ of a matrix $A=\left(a_{\kappa \lambda}\right)$ so that the ordinary rule $\left(A_{1} A_{2}\right)^{\prime}=A_{2}^{\prime} A_{1}^{\prime}$ holds for any two matrices whose product is defined. We must take $A^{\prime}$ not as a matrix with coefficients in $K$ but in the antisymmetric division ring $K^{\prime}$. This $K^{\prime}$ consists of all symbols $\alpha^{\prime}$ where $\alpha$ is an arbitrary element of $K$. We have $\alpha^{\prime}=\beta^{\prime}$, if and only if $\alpha=\beta$, and we define addition and multiplication by

$$
\alpha_{1}^{\prime}+\alpha_{2}^{\prime}=\left(\alpha_{1}+\alpha_{2}\right)^{\prime} ; \quad \alpha_{1}^{\prime} \alpha_{2}^{\prime}=\left(\alpha_{2} \alpha_{1}\right)^{\prime} .
$$

If we now set $A^{\prime}=\left(a_{\lambda_{\kappa}}^{\prime}\right)$ ( $\kappa$, row-index; $\lambda$, column-index), we readily obtain $\left(A_{1} A_{2}\right)^{\prime}=A_{2}^{\prime} A_{1}^{\prime}$.

4. The irReducible constituents of a set of square matrices

1. Consider a set $及$ of elements $\alpha$ of any kind, and a number of sets of matrices $\mathfrak{A}, \mathfrak{B}, \cdots$. We assume that to every $\alpha$ in $\mathbb{B}$ there corresponds a matrix $A_{\alpha}$ in $\mathfrak{A}$, a matrix $B_{\alpha}$ in $\mathfrak{B}$, etc., such that all the matrices of $\mathfrak{A}, \mathfrak{B}, \cdots$ appear at least once in the form $A_{\alpha}, B_{\alpha}, \cdots$ respectively. We then say that $\mathfrak{A}, \mathfrak{B}, \cdots$ are related sets. Equations between related sets $\mathfrak{A}, \mathfrak{B}, \mathfrak{C}, \mathfrak{D}$ such as

$$
\mathfrak{A}=\mathfrak{B}, \quad \mathfrak{A}=\left(\begin{array}{ll}
\mathfrak{B} & \\
\mathfrak{C} & \mathfrak{D}
\end{array}\right), \quad \mathfrak{A} P=P \mathfrak{B}(\text { with a fixed matrix } P \text { ) }
$$

indicate that for every $\alpha$ in $\$$ the corresponding equations hold:

$$
A_{\alpha}=B_{\alpha}, \quad A_{\alpha}=\left(\begin{array}{ll}
B_{\alpha} & \\
C_{\alpha} & D_{\alpha}
\end{array}\right), \quad A_{\alpha} P=P B_{\alpha} .
$$

2. Let $\mathfrak{A}$ be a set of square matrices $A$ of degree $n$ interpreted as linear transformations $X \rightarrow X^{*}=A X$ of an $n$-dimensional vector space $\mathfrak{B}$. If we introduce new coordinates by a linear transformation $x_{\kappa}=\sum p_{\kappa} z_{\lambda}$, the set $\mathfrak{A}$ is replaced by $P^{-1} \mathfrak{U} P$. These two sets $\mathfrak{A}$ and $P^{-1 \mathfrak{A} P}$ are similar, $\mathfrak{A} \sim P^{-1} \mathfrak{A} P$; they 
are related (with $\mathfrak{Z}=\mathfrak{U}$ ). Similar sets of ten are considered as not essentially different.

The vectors $X$ of $\mathfrak{B}$ form an additive abelian group, and we can now introduce two kinds of operators: As the first kind of operator, we take the elements $\rho$ of $K$, the operation being defined as $r$-multiplication of $X$ by $\rho$ (as before). As the second kind of operator, we take the elements $\alpha$ of $\not$, the operation being defined by $\alpha X=A_{\alpha} X$.

Let $\mathfrak{B}$ be a second set of matrices which is related to $\mathfrak{A}$, and let $\mathfrak{W}$ be a vector space in which the corresponding linear transformations take place. Then $\mathfrak{A} \sim \mathfrak{B}$, if and only if $\mathfrak{B}$ and $\mathfrak{B}$ are operator-isomorphic (with regard to $\mathfrak{Z}$ and $K)$.

More generally, let us assume that we have an operator-homomorphic mapping of $\mathfrak{B}$ upon an admissible subgroup $\mathfrak{B}_{0}$ of $\mathfrak{B}$. This mapping is given by a linear transformation $Y \rightarrow X=P Y$, ( $Y$ in $\mathfrak{B}, X$ in $\mathfrak{B})$. The condition for an operator-homomorphism with regard to 3 , then, is $\alpha X=P(\alpha Y)$ for every $\alpha$ in 3 , i.e., $A_{\alpha} P Y=P B_{\alpha} Y$. Since this must hold for every $Y$ in $\mathfrak{W}$, we find

$$
\mathfrak{U} P=P \mathfrak{B} \text {. }
$$

We then say that $P$ intertwines $\mathfrak{A}$ and $\mathfrak{B}$. When $\mathfrak{A}$ and $\mathfrak{B}$ are replaced by similar sets $M^{-1} \mathfrak{Q} M$ and $N^{-1} \mathfrak{B} N$, we have

$$
\left(M^{-1 \mathfrak{A} M}\right)\left(M^{-1} P N\right)=\left(M^{-1} P N\right)\left(N^{-1} \mathfrak{B} N\right)\left({ }^{15}\right)
$$

and the matrix $M^{-1} P N$ obviously takes the place of $P$.

3 . If the group $\mathfrak{B}$ with the sets of operators $\mathfrak{B}, K$ is simple, then $\mathfrak{A}$ is an irreducible set. If $\mathfrak{Q}$ is reducible, $\mathfrak{B}$ has an admissible subgroup $\tilde{\mathfrak{B}}$ with $\mathfrak{B} \supset \tilde{\mathfrak{B}} \supset\{0\}$. This $\tilde{\mathfrak{B}}$, then, is a linear subspace which is invariant under the transformations of $\mathfrak{A}$. If we choose the basis of $\mathfrak{B}$ such that the last $r$ basis elements form a basis of $\tilde{\mathfrak{B}}$, then $\mathfrak{A}$ splits in the form

$$
\mathfrak{A}=\left(\begin{array}{ll}
\mathfrak{M}_{1} & \\
\mathfrak{M}_{3} & \mathfrak{M}_{4}
\end{array}\right)
$$

where $\mathfrak{A}$ is broken up according to the scheme $(n-r, r \mid n-r, r)$. Conversely if $\mathfrak{A}$ has this form with regard to a suitable coordinate system, then $\mathfrak{A}$ is reducible. Here $\mathfrak{M}_{4}$ are the transformations induced by $\mathfrak{A}$ in $\tilde{\mathfrak{B}}$, and $\mathfrak{M}_{1}$ are the transformations induced by $\mathfrak{A}$ in $\mathfrak{B} / \tilde{\mathfrak{B}}$.

We may interpret the matrices of $\mathfrak{A}$ by means of linear transformations $U \rightarrow U^{*}=U A$ of a contragredient vector space $\mathfrak{W}$. If $\mathfrak{A}$ splits in the form (9), then $\mathfrak{W}$ has an invariant subspace $\tilde{\mathfrak{B}}$ of $n-r$ dimensions, and the transformations of $\mathfrak{A}$ induce the transformations of $\mathfrak{M}_{1}$ in $\tilde{\mathfrak{B}}$ and those of $\mathfrak{M}_{4}$ in $\mathfrak{W} / \tilde{\mathfrak{W}}$, so that the roles of $\mathfrak{M}_{1}$ and $\mathfrak{M}_{4}$ are interchanged.

4. We now consider a composition series of $\mathfrak{B}$

(15) Schur [26]. 


$$
\mathfrak{B}=\mathfrak{B}_{0} \supset \mathfrak{B}_{1} \supset \mathfrak{B}_{2} \supset \ldots \supset \mathfrak{B}_{r}=\{0\} .
$$

Let $E_{\nu}^{(i)}\left(\nu=1,2, \cdots, a_{i}\right)$ be a maximal set of vectors of $\mathfrak{B}_{i-1}$ which are $r$-independent $\left(\bmod \mathfrak{B}_{i}\right)$. Then the totality of all vectors

$$
E_{1}^{(i)} z_{1}+E_{2}^{(i)} z_{2}+\cdots+E_{a}^{(i)} z_{a i}, \quad z_{\lambda} \text { in } K,
$$

form a complete residue system $\mathfrak{P}_{i}$ of $\mathfrak{B}_{i-1}\left(\bmod \mathfrak{B}_{i}\right)$. All the vectors $E_{\nu}^{(i)}$, arranged according to increasing $i$ form a basis of $\mathfrak{B}$, and with regard to this basis, $\mathfrak{A}$ has the form

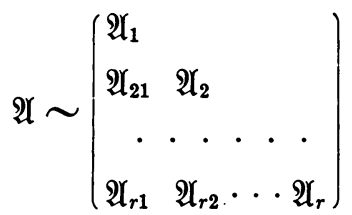

where $\mathfrak{A}_{i}$ is an irreducible set of square matrices of degree $a_{i}$. These $\mathfrak{A}_{i}$ are called the irreducible constituents of $\mathfrak{A}$.

From Jordan-Hölder's theorem, we obtain at once $\left({ }^{16}\right)$

(4.4A) The irreducible constituents of a set $\mathfrak{A}$ of square matrices are uniquely determined apart from their arrangement, if similar sets are considered as equal.

When we replace the $E_{\lambda}^{(i)}$ by another basis of $\mathfrak{B}_{i-1}\left(\bmod \mathfrak{B}_{i}\right)$, then $\mathfrak{A}_{i}$ is replaced by a similar set. We obtain this new form of $\mathfrak{A}$ by a similarity transformation of type (3.3C).

If a formula (11) holds where each $\mathfrak{A}_{i}$ is a reducible or irreducible set of square matrices of some degree $a_{i}$, we say that each $\mathfrak{A}_{i}$ is a constituent of $\mathfrak{A}$. In particular, we call $\mathfrak{A}_{1}$ a top constituent and $\mathfrak{A}_{r}$ a bottom constituent.

Let $\mathfrak{A}$ and $\mathfrak{B}$ again be two related intertwined sets, $\mathfrak{A} P=P \mathfrak{B}$ and $P \neq 0$. We consider again the mapping of $\mathfrak{B}$ upon a certain admissible subgroup $\tilde{\mathfrak{B}}$ of $\mathfrak{B}$ which is defined by $P$. The vectors of $\mathfrak{B}$ which are mapped upon 0 form an admissible subgroup $\tilde{\mathfrak{B}}$ of $\mathfrak{W}$, and we have $\mathfrak{B} \simeq \mathfrak{W} / \mathfrak{\mathfrak { B }}$. If we use these subgroups in order to split $\mathfrak{A}$ and $\mathfrak{B}$, we have with regard to suitable coordinate systems

$$
\mathfrak{A}=\left(\begin{array}{cc}
* & 0 \\
* & \mathfrak{U}
\end{array}\right), \quad P=\left(\begin{array}{cc}
0 & 0 \\
I & 0
\end{array}\right), \quad \mathfrak{B}=\left(\begin{array}{cc}
\mathfrak{U} & 0 \\
* & *
\end{array}\right)
$$

This gives Schur's lemma $\left({ }^{17}\right)$. [11].

${ }^{(16)}$ This simple proof for the uniqueness of the irreducible constituents is due to W. Krull

(17) I. Schur [26]. Schur's proof is extremely simple. By means of (7), similarity transformations of $\mathscr{A}$ and $\mathfrak{B}$ are performed such that $P$ assumes the desired form, and then $\mathscr{A}$ and $\mathfrak{B}$ must have the form given here. 
(4.4B) If two related sets $\mathfrak{A}$ and $\mathfrak{B}$ are intertwined by a matrix $P \neq 0$, then there exists a bottom constituent of $\mathfrak{A}$ which appears as a top constituent of $\mathfrak{B}$. If $\mathfrak{A}$ and $\mathfrak{B}$ are irreducible, then $P$ is nonsingular, and $\mathfrak{A}$ and $\mathfrak{B}$ are similar.

5 . We now apply the results of Section 1 . We choose the residue systems of $\mathfrak{B}_{i-1} / \mathfrak{B}_{i}$ always as in (10), consisting of all linear combinations of some $r$-independent vectors. From (1.1B) we see that the results of Section 1 remain valid, if we restrict the choice of residue systems by this condition.

Any change of the residue system $\mathfrak{B}_{j}$ as used in Section 1 can be accomplished by a succession of changes of the following kind: The elements of $\mathfrak{B}_{j}$ are multiplied by elements of some $\mathfrak{B}_{i}$, with $i>j$. This now corresponds to replacing $E_{\nu}^{(j)}$ by $E_{\nu}^{(j)}+S_{\nu}^{(i)}$ where each $S_{\nu}^{(i)}$ is of the form (10). This basis transformation corresponds to the linear transformation $X_{\kappa}^{*}=X_{\kappa}$ for $\kappa \neq i$, $X_{i}^{*}=X_{i}-Q X_{j}$ where the vector $X$ is broken up according to the scheme $\left(a_{1}, \cdots, a_{k} \mid 1\right)$ and the matrix $Q$ of type $\left(a_{i}, a_{j}\right)$ is formed by the components $z_{\lambda},(10)$, of the vectors $S_{\nu}^{(i)}$. This is a transformation $T_{i j}(-Q)=T_{i j}(Q)^{-1}$ (cf. §3.3), and $\mathfrak{A}$ is there replaced by $T_{i j}(Q)^{-1} \mathfrak{A} T_{i j}(Q)$. According to (3.3A), we have to add the $i$ th column in (11), $r$-multiplied by $Q$, to the $j$ th column, and the $j$ th row, $l$-multiplied by $-Q$, to the $i$ th row. Because of $i>j$, the triangular form (11) of $\mathfrak{A}$ is not disturbed:

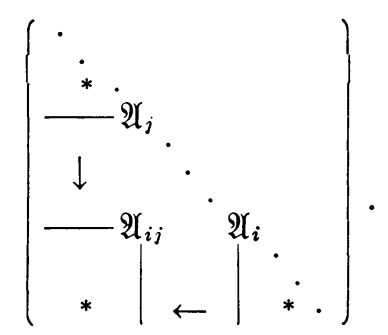

Only the sets $\mathfrak{A}_{i \lambda}$ with $\lambda \leqq j$ and $\mathfrak{A}_{\mu j}$ with $\mu \geqq i$ will be changed. We denote such a special similarity transformation of $\mathfrak{A}$ as an elementary similarity transformation of $\mathfrak{A}$. All the $\mathfrak{A}_{\kappa}$ remain unchanged.

Consider again two related sets of square matrices $\mathfrak{A}$ and $\mathfrak{B}$, operating in the vector spaces $\mathfrak{B}$ and $\mathfrak{W}$ respectively. We assume that both split into irreducible constituents

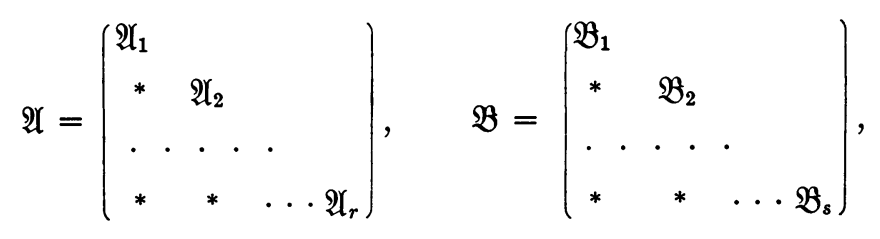

where $\mathfrak{A}_{\rho}$ has the degree $a_{\rho}$ and $\mathfrak{B}_{\sigma}$ has the degree $b_{\sigma}$. If $P$ is an intertwining matrix, we break up $P$ according to the scheme $\left(a_{1}, \cdots, a_{r} \mid b_{1}, \cdots, b_{s}\right)$; say 
$P=\left(P_{\kappa \lambda}\right)$. Then the products $\mathfrak{A} P$ and $P \mathfrak{B}$ can be obtained in the ordinary manner $(\$ 3.3)$. We say, therefore, that the intertwining matrix $P$ has been broken $u p$ in accordance with the splitting of $\mathfrak{A}$ and $\mathfrak{B}$ in (12). Application of (1.1A) to the homomorphic mapping of $\mathfrak{W}$ upon a subgroup of $\mathfrak{B}$ then yields

(4.5A) Let $\mathfrak{A}$ and $\mathfrak{B}$ be two related sets of square matrices which split into irreducible constituents (12), and let $P$ be an intertwining matrix. We can apply to $\mathfrak{A}$ and $\mathfrak{B}$ a succession of elementary similarity transformations such that the matrix $P^{*}$ which afterwards takes the place of $P(c f . \S 4.2)$ contains in each row and each column at most one term not equal to 0 , if broken up in accordance with the splitting of $\mathfrak{A}$ and $\mathfrak{B}$.

If $P^{*}=\left(P_{\kappa \lambda}^{*}\right)$, then $\mathfrak{A}_{\kappa} P_{\kappa \lambda}^{*}=P_{\kappa \lambda}^{*} \mathfrak{B}_{\lambda}$ because of this form of $P^{*}$. If $P_{\kappa \lambda}^{*} \neq 0$, then $P_{\kappa \lambda}$ is nonsingular, according to (4.4B). Since for a given $\lambda$ this may occur for at most one value of $\kappa$, after a succession of similarity transformations of type (3.3C), each $P_{k \lambda}^{*}$ is either 0 or a unit matrix.

Assume now that $P$ is nonsingular so that $\mathfrak{A}$ and $\mathfrak{B}$ are similar. Then every row of $P^{*}$ must contain one $P_{k \lambda}^{*} \neq 0$, say for instance $P_{1 j}^{*} \neq 0$. We denote the sets similar to $\mathfrak{A}$ and $\mathfrak{B}$, which we have obtained by $\mathfrak{A}$ and $\mathfrak{B}$ again, and use the notation (12). Then it easily follows from $\mathfrak{A} P^{*}=P^{*} \mathfrak{B}$ by forming the first rows of the products that

$$
0=\mathfrak{B}_{j 1}, 0=\mathfrak{B}_{j 2}, \cdots, 0=\mathfrak{B}_{j, j-1}, \mathfrak{A}_{1}=\mathfrak{B}_{j} .
$$

We replace $\mathfrak{B}$ by the similar set $Z_{j-1, j}^{-1} \mathfrak{B} Z_{j-1, j}$ (cf. (3.3B)). Because $\mathfrak{B}_{j, j-1}=0$, the triangular form (12) of $\mathfrak{B}$ is not disturbed,

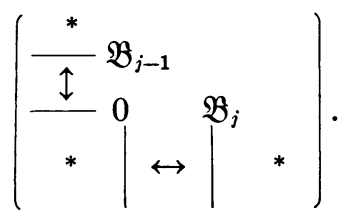

The irreducible constituents of $\mathfrak{B}$ remain the same, only $\mathfrak{B}_{j-1}$ and $\mathfrak{B}_{j}$ are interchanged. Such a similarity transformation of $\mathfrak{B}$ is an admissible permutation of rows and columns which can always be applied, if $\mathfrak{B}_{j, j-1}=0$. According to $\$ 4.2, P^{*}$ must be replaced by $P^{*} Z_{j-1, j}$, i.e., the columns $j-1$ and $j$ are to be interchanged (3.3B); but the essential properties of $P^{*}$ are not destroyed. Similarly, we can interchange $\mathfrak{B}_{j}$ with $\mathfrak{B}_{j-2}, \mathfrak{B}_{j-3}, \cdots, \mathfrak{B}_{1}$. The matrix $P^{* *}$ which takes the place of $P$ will have the first row $(I, 0, \cdots, 0)$. We now work with the second row of $P^{* *}$. The element $P_{2 k}^{* *}=I$ in it will not stand in the first column. After a number of further admissible permutations of rows and columns, we may bring it into the second column. Continuing in this manner, we will finally replace $P$ by $I$. This gives (cf. (1.2A))

(4.5B) If $\mathfrak{A}$ and $\mathfrak{B},(12)$, are two similar sets of square matrices which break 
up into irreducible constituents, then it is possible to carry $\mathfrak{B}$ into $\mathfrak{A}$ by a succession of similarity transformations of types (3.3A), (3.3B), and $(3.3 \mathrm{C})\left({ }^{18}\right)$.

\section{The Loewy constituents}

1. A set $\mathfrak{A}$ of square matrices of degree $n$ is completely reducible, if the corresponding vector space $\mathfrak{B}$ (with $\mathfrak{A}$ and $K$ as sets of operators) is completely reducible. If we choose the composition series of $\mathfrak{B}$ and the $\mathfrak{B}_{i}$ as in $\$ 2.1$, then the formula (11) takes the form

$$
\mathfrak{A} \sim\left(\begin{array}{llll}
\mathfrak{A}_{1} & & & \\
& \mathfrak{A}_{2} & & \\
& & \cdot & \\
& & & \mathfrak{A}_{r}
\end{array}\right), \quad \mathfrak{F}_{\rho} \text { irreducible, }
$$

with zeros above and below the main diagonal. Conversely, if such a formula holds, then $\mathfrak{A}$ is completely reducible.

In the general case, let

$$
\mathfrak{B}=\mathfrak{M}_{0} \supset \mathfrak{M}_{1} \supset \mathfrak{M}_{2} \supset \cdots \supset \mathfrak{M}_{t}=\{0\}
$$

be a Loewy series for $\mathfrak{B}$. If we choose the basis of $\mathfrak{B}$ by first taking a maximal set of vectors of $\mathfrak{M}_{0}$ which are $r$-independent $\left(\bmod \mathfrak{M}_{1}\right)$, then a maximal set of vectors of $\mathfrak{M}_{1}$ which are $r$-independent $\left(\bmod \mathfrak{M}_{2}\right)$, etc., then $\mathfrak{A}$ has the form

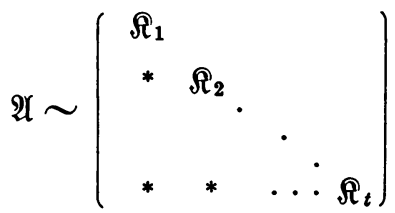

and each $\mathfrak{R}_{\lambda}$ is completely reducible, since $\mathfrak{M}_{\lambda-1} / \mathfrak{M}_{\lambda}$ is completely reducible. We say that $\mathfrak{A}$ here appears in a Loewy form; every Loewy form of $\mathfrak{A}$ is obtained from a Loewy series of $\mathfrak{B}$. Two Loewy forms are of special importance, the lower and the upper Loewy form $\left({ }^{19}\right)$, corresponding to the lower and upper Loewy series of $\mathfrak{B}$, both having the same length (cf. (2.4A)) which will be denoted by $L=L(\mathfrak{A})$. We write them:

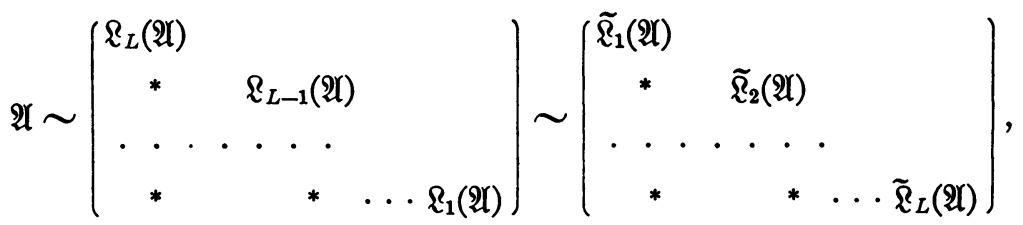

(18) These transformations are to be applied to the form (12) of $\mathfrak{Y}$ and $\mathfrak{B}$.

$\left.{ }^{19}\right)$ Cf. A. Loewy $[14,15]$, W. Krull [12], B. L. van der Waerden [29]. 
where the first is the lower and the second is the upper Loewy form. The lower Loewy constituents $\mathfrak{R}_{1}(\mathfrak{A}), \mathfrak{R}_{2}(\mathfrak{A}), \cdots$ are numerated starting from the bottom, and the upper Loewy constituents $\tilde{\mathfrak{R}}_{1}(\mathfrak{H}), \tilde{\mathfrak{R}}_{2}(\mathfrak{H}), \cdots$ starting from the top.

The constituent $\mathfrak{R}_{1}(\mathfrak{H})$ is the maximal completely reducible set which can appear as bottom constituent of $\mathfrak{A}$. If $\mathfrak{A}$ splits into $\mathfrak{B}$ and $\mathfrak{R}_{1}(\mathfrak{U})$, then

$$
\mathfrak{R}_{i+1}(\mathfrak{H}) \sim \mathfrak{R}_{i}(\mathfrak{B}) .
$$

Similarly, $\tilde{\mathfrak{R}}_{1}(\mathfrak{A})$ is the maximal completely reducible set which can appear as a top constituent of $\mathfrak{A}$; and if $\mathfrak{A}$ splits into $\tilde{\mathfrak{Q}}_{1}(\mathfrak{H})$ and $\mathfrak{\mathfrak { B }}$, then

$$
\widetilde{\mathfrak{R}}_{i+1}(\mathfrak{A}) \sim \mathfrak{R}_{i}(\tilde{\mathfrak{B}}) .
$$

The transformations of $\mathfrak{A}$ transform the space $\mathfrak{M}_{i-1} / \mathfrak{M}_{j},(j \geqq i)$, into a part of itself and induce, therefore, a set of linear transformations in the space. This set is obtained from (13) by removing the rows and columns with an index less than $i$ or greater than $j$. We denote this set by $\Re(i \cdots j)$; its main diagonal starts with $\Omega_{i}$ and ends with $\Omega_{j}$. Since in the case of the lower and the upper Loewy series the groups $\mathfrak{M}_{h}$ are uniquely determined, we have

(5.1A) The constituent $\mathfrak{R}(i \cdots j)$ of the lower Loewy normal form is uniquely determined apart from similarity transformation. The corresponding fact holds for the upper Loewy normal form.

From (2.4B), we obtain

(5.1B) The Loewy constituents $\mathfrak{R}_{i}(\mathfrak{A})$ and $\mathfrak{R}_{L-i}(\mathfrak{H})(L=L(\mathfrak{A}) ; i=1,2, \cdots, L)$ have at least one common irreducible constituent.

2. Application of the theorems (2.2A) and (2.3A) gives

(5.2A) Let $\mathfrak{A}$ and $\mathfrak{B}$ be two related sets of square matrices, both written in Loewy form

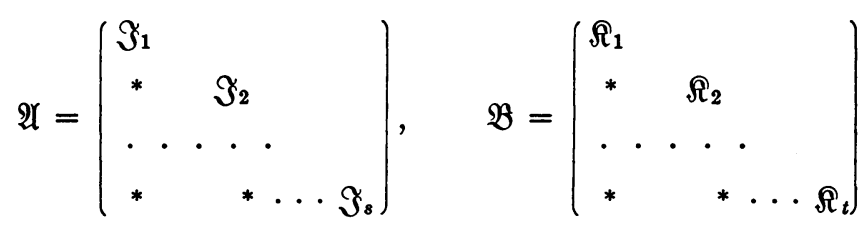

$\left(\Im_{\sigma}\right.$ and $\Re_{\tau}$ completely reducible). Let $P=\left(P_{\kappa \lambda}\right)$ be an intertwining matrix broken up in accordance with the splitting (15) of $\mathfrak{A}$ and $\mathfrak{B}(c f . \S 4.5)$. ( $\alpha)$ If $\mathfrak{A}$ is in its lower Loewy normal form, then $P_{\kappa \lambda}=0$ for $s-\kappa>t-\lambda$. ( $\left.\beta\right)$ If $\mathfrak{B}$ is in its upper Loewy normal form, then $P_{\kappa \lambda}=0$ for $\kappa<\lambda$.

In other words: In the case $(\alpha), P$ has the form given in $(16 \alpha)$ below; if $s>t$, the first $s-t$ rows in $P$ consist of zeros. In the case $(\beta), P$ has the form 
$(16 \beta)$; for $s<t$, the last $t-s$ columns consist of zeros:

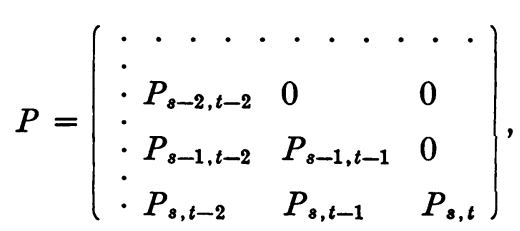

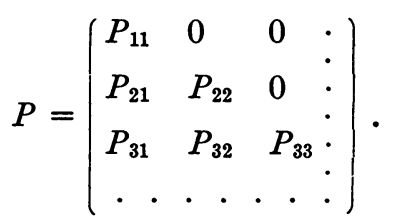

3. When a set $\mathfrak{A}$ is given in the form (11), splitting into irreducible constituents, we can use the method of $\$ 2.5$ in order to determine the Loewy constituents $\mathfrak{R}(\mathfrak{A})$. We consider one constituent $\mathfrak{A}_{i}$ in $\mathfrak{A}$,

$$
\mathfrak{A}=\left(\begin{array}{lll}
* & & \\
* & \mathfrak{A}_{i} & \\
* & \mathfrak{S} & \mathfrak{D}
\end{array}\right),
$$

where the rows and columns $i+1, i+2, \cdots, r$ of (11) are grouped together in $\mathfrak{D}$. If (11) belongs to the composition series $\mathfrak{B}, \mathfrak{B}_{1}, \mathfrak{B}_{2}, \cdots, \mathfrak{B}_{r}$ and $\mathfrak{B}_{\tau}$ is a complete residue system of $\mathfrak{B}_{\tau-1}\left(\bmod \mathfrak{B}_{\tau}\right)$, then the question is whether we can change $\mathfrak{B}_{i}$ so that it forms an admissible subgroup. The only freedom which we have is that we can add arbitrary vectors of $\mathfrak{B}_{i}$ to the basis elements of $\mathfrak{B}_{i}$. This amounts to an elementary similarity transformation of (17), involving the second and third row and column (cf. \$4.5). If after the change $\mathfrak{B}_{i}$ is an admissible subgroup, then $\mathbb{S}$ must become 0 , since the modified $\mathfrak{P}_{i}$ are invariant under $\mathfrak{A}$. But an elementary similarity transformation replaces $\mathfrak{E}$ by $\left(\mathfrak{S}+\mathfrak{D} Q-Q \mathfrak{A}_{i}\right.$; so that the residue system $\mathfrak{B}_{i}$ will be of the lowest kind, if and only if this is 0 for a suitable $Q$, and $\mathfrak{A}_{i}$ will belong to $L_{1}(\mathfrak{U})$. Hence

(5.3A) The first Loewy constituent $\mathfrak{l}_{1}(\mathfrak{H})$ consists of those irreducible constituents $\mathfrak{A}_{i},(15)$, for which a matrix $Q$ can be determined such that in (17) $\mathfrak{C}=Q \mathfrak{A}_{i}-\mathfrak{D} Q$.

After similarity transformations, we may assume that all $\mathfrak{A}_{i}$ of this type stand in columns in which otherwise only zeros appear. In order to find $\mathfrak{R}_{2}(\mathfrak{A})$ we have to remove the rows and columns of the $\mathfrak{A}_{i}$ "of lowest kind" from $\mathfrak{A}$, and treat the remaining set $\mathfrak{B}$ in the same manner; we have $\mathfrak{R}_{\nu+1}(\mathfrak{A})=\mathfrak{R}_{\nu}(\mathfrak{B})$.

Moving all the constituents $\mathfrak{A}$ of lowest kind to the bottom by admissible permutations $\$ 4.5, \mathfrak{l}_{1}(\mathfrak{U})$ will appear at the bottom of $\mathfrak{A}$. After removing its rows and columns from $\mathfrak{A}$ and treating the remainder in the same fashion, we 
finally arrive at the lower Loewy form of $\mathfrak{A}$. It is remarkable in this connection that the criterion (5.3A) only depends on the solution of linear equations for the coefficients of the matrix $Q$.

4. The dualism between the upper and lower Loewy form can be realized in the following manner. We replace every matrix $A$ of $\mathfrak{A}$ by its transposed $A^{\prime}$, $\S 3.5$. If $\mathfrak{A}$ is in its lower normal form, (14), the new set $\mathfrak{A}^{\prime}$ formed by all $A^{\prime}$ will have the following form

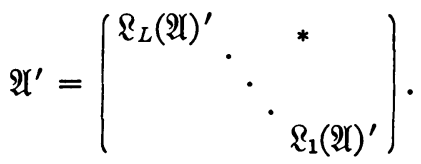

If we arrange the rows and columns in reverse order, $\mathfrak{U}^{\prime}$ splits into the constituents $\mathfrak{R}_{1}(\mathfrak{H})^{\prime}, \cdots, \mathfrak{R}_{L}(\mathfrak{R})^{\prime}$. In this manner, we easily see that

$$
\mathfrak{R}_{\nu}(\mathfrak{X})^{\prime}=\mathfrak{I}_{\nu}\left(\mathfrak{X}^{\prime}\right)\left(\nu=1,2, \cdots, L ; L=L(\mathfrak{X})=L\left(\mathfrak{A}^{\prime}\right)\right) .
$$

Using this method, we can derive results concerning the upper Loewy form from those concerning the lower Loewy form in $\$ 5.3$.

\section{AdDitional Remarks}

1. We consider two related sets $\mathfrak{A}$ and $\mathfrak{B}$ of matrices which split completely in to irreducible constituents, i.e.,

$$
\mathfrak{U}=\left(\begin{array}{lllll}
\mathfrak{A}_{1} & & & & \\
& \mathfrak{A}_{2} & & & \\
& & \cdot & & \\
& & & \cdot & \\
& & & \mathfrak{A}_{r}
\end{array}\right), \quad \mathfrak{B}=\left(\begin{array}{lllll}
\mathfrak{B}_{1} & & & & \\
& \mathfrak{B}_{2} & & \\
& & \cdot & \\
& & & \cdot \\
& & & \mathfrak{B}_{8}
\end{array}\right) .
$$

If $P$ is an intertwining matrix, $\mathfrak{A} P=P \mathfrak{B}$, we break up $P$ according to this splitting, $P=\left(P_{\kappa \lambda}\right)$ (cf. $\left.\$ 4.5\right)$. The condition for $P_{\kappa \lambda}$ becomes $\mathfrak{A}_{\kappa} P_{\kappa \lambda}=P_{\kappa \lambda} \mathfrak{B}_{\lambda}$. Using Schur's lemma, we obtain

(6.1A) Let $\mathfrak{A}$ and $\mathfrak{B}$ be two related sets of matrices which split completely into irreducible constituents $\mathfrak{A}_{1}, \mathfrak{A}_{2}, \cdots, \mathfrak{A}_{r}$ and $\mathfrak{B}_{1}, \mathfrak{B}_{2}, \cdots, \mathfrak{B}_{8}$ respectively. If $P=\left(P_{\kappa \lambda}\right)$ is an intertwining matrix broken up in accordance with the splitting of $\mathfrak{A}$ and $\mathfrak{B}$, then either $P_{\kappa \lambda}=0$, or $\mathfrak{A}_{\kappa} \sim \mathfrak{B}_{\lambda}$ and $P_{\kappa \lambda}$ is nonsingular and intertwines $\mathfrak{A}_{\kappa}$ and $\mathfrak{B}_{\lambda}$. Conversely, if these conditions are satisfied $P=\left(P_{\kappa \lambda}\right)$ intertwines $\mathfrak{A}$ and $\mathfrak{B}$.

2. The matrices $P$ which intertwine a set $\mathfrak{A}$ of square matrices with itself, $\mathfrak{A} P=P \mathfrak{A}$, form a ring, the commuting ring $\mathfrak{S}(\mathfrak{U})$ of $\mathfrak{A}$. If $P$ in $\mathfrak{S}(\mathfrak{A})$ is a nonsingular matrix, then $P^{-1}$ also belongs to $\mathfrak{S}(\mathfrak{A})$. From Schur's lemma, we find that

(6.2A) The commuting ring of an irreducible set is a division ring. 
Denote by $k \times \mathfrak{A}$ the set which splits completely into $k$ equal constituents $\mathfrak{A}$, and by $[\mathfrak{A}]_{k}$ the set of all matrices $\left(A_{\kappa \lambda}\right)$ of degree $k$ in which the $A_{\kappa \lambda}$ are arbitrary elements of $\mathfrak{A}$. I.e.,

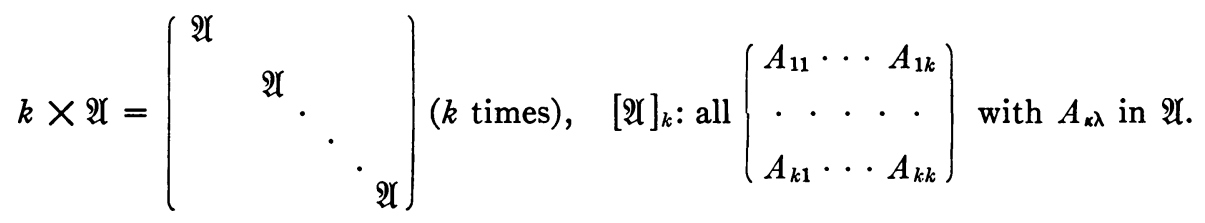

We then state

(6.2B) $(\alpha) \mathbb{S}(k \times \mathfrak{A})=[\mathfrak{G}(\mathfrak{A})]_{k}$. ( $\left.\beta\right)$ If $\mathfrak{A}$ contains 0 and $I$, then $\mathfrak{E}\left([\mathfrak{A}]_{k}\right)$ $=k \times \mathfrak{S}(\mathfrak{A}) .(\gamma) \mathfrak{S}(\mathfrak{S}(k \times \mathfrak{A}))=k \times \mathfrak{S} \mathfrak{S}(\mathfrak{A})$.

Proof. $(\alpha)$ follows at once from (6.1A). In the case of $(\beta)$, let $P$ be a matrix of $\mathfrak{S}\left([\mathfrak{A}]_{k}\right)$ and set $P=\left(P_{\kappa \lambda}\right)$ where all the $P_{\kappa \lambda}$ have the degree $a$ of $\mathfrak{A}$. We first choose all $A_{\kappa \lambda}=0$ except one, say $A_{\rho \sigma}$. From $\left(A_{\kappa \lambda}\right)\left(P_{\kappa \lambda}\right)=\left(P_{\kappa \lambda}\right)\left(A_{\kappa \lambda}\right)$, it follows that $A_{\rho \sigma} P_{\sigma \lambda}=0$ for $\lambda \neq \sigma, A_{\rho \sigma} P_{\sigma \sigma}=P_{\rho \rho} A_{\rho \sigma}$. Taking first $A_{\rho \sigma}=I_{r}$, and then taking $\rho=\sigma$ and taking $A_{\rho \rho}$ arbitrarily, we obtain $(\beta)$. The statement $(\gamma)$ is obtained from $(\alpha)$ by applying $(\beta)$ to $\mathfrak{S}(\mathfrak{A})$ instead of $\mathfrak{A}$; the matrices 0 and $I$ belong to $\mathfrak{S}(\mathfrak{A})$.

From (6.1A) and $(6.2 \mathrm{~B} \alpha)$ also follows

(6.2C) If $\mathfrak{A}$ splits completely into $k_{1} \times \mathfrak{A}_{1}, \cdots, k_{r} \times \mathfrak{A}_{r}$, where $\mathfrak{A}_{1}, \mathfrak{A}_{2}, \cdots, \mathfrak{A}_{r}$ are irreducible and not similar, then $\mathfrak{S}(\mathfrak{A})$ splits completely into $\left[\mathfrak{S}\left(\mathfrak{A}_{1}\right)\right]_{k_{1}}$, $\left[\mathfrak{S}\left(\mathfrak{A}_{2}\right)\right]_{k_{2}}, \cdots,\left[\mathfrak{S}\left(\mathfrak{A}_{r}\right)\right]_{k_{r}}$.

The $\Subset\left(\mathfrak{A}_{\kappa}\right)$ here may be reducible or irreducible (see $\$ 9.3$ below).

In the general case, a structure theory of the ring $\mathfrak{S}(\mathfrak{H})$ is contained as a special case in the results of Fitting $\left({ }^{20}\right)$.

3. With regard to $[\mathfrak{A}]_{k}$, we can prove

(6.3A) If $\mathfrak{A}$ is reducible, so is $[\mathfrak{H}]_{k}$. If $\mathfrak{A}$ is irreducible and contains 0 without consisting of the zero matrix, then $[\mathfrak{U}]_{k}$ is irreducible.

Proof. If $\mathfrak{A}$ is reducible, we may assume that it splits into two constituents, i.e.,

$$
\mathfrak{A}=\left(\begin{array}{ll}
\Omega_{1} & \\
\Omega_{3} & \Omega_{4}
\end{array}\right) .
$$

Writing every $A_{\kappa \lambda}$ in the corresponding form, $\left(A_{\kappa \lambda}\right)$ appears as a matrix of degree $2 k$. We rearrange the rows and columns, first taking those with an odd index and then those with an even index. After this similarity transformation, $[\mathfrak{A}]_{k}$ will split.

${ }^{(20)}$ Fitting [8]. 
If $\mathfrak{A}$ satisfies the assumptions of the second part of $(6.3 \mathrm{~A})$, and if $[\mathfrak{A}]_{k}$ were reducible, then $[\mathfrak{T}]_{k}$ also would be reducible, where $\mathfrak{T}$ is the ring generated by $\mathfrak{A}$. That this is not so can be easily seen from a simple argument of Weyl $\left({ }^{21}\right)$.

4. Next, we prove an extension of a theorem of A. H. Clifford( $\left.{ }^{22}\right)$

(6.4A) Let $\mathfrak{B}$ be a set of matrices of degree $b$ and denote by $\mathfrak{S}$ the set of all matrices $P$ of degree $b$ for which $\mathfrak{B P}$ and $P \mathfrak{B}$ consist of the same matrices. The total number of irreducible constituents of $\mathfrak{S}$ is at least equal to the number $L(\mathfrak{B})$ of Loewy constituents of $\mathfrak{B}, \S 5.1$.

Proof. After a similarity transformation of $\mathfrak{B}$, we may assume that $\mathfrak{B}$ appears in its lower Loewy normal form. Let $P$ be a fixed element of $\mathfrak{S}$. We form the set 3 of all pairs $\left(B_{1}, B_{2}\right)$ of two elements $B_{1}, B_{2}$ of $\mathfrak{B}$ for which $B_{1} P=P B_{2}$. To every element of $\$$ there corresponds a first matrix $B_{1}$ and a second matrix $B_{2}$. We thus obtain two related sets $\mathfrak{B}_{1}$ and $\mathfrak{B}_{2}$ such that $\mathfrak{B}_{1} P=P \mathfrak{B}_{2}$. Since $\mathfrak{B}_{1}$ and $\mathfrak{B}_{2}$ both consist of the same matrices as $\mathfrak{B}$, both are in their Loewy normal form. We can now apply (5.2A). Since $\mathfrak{B}_{1}$ and $\mathfrak{B}_{2}$ both have $L(\mathfrak{B})$ Loewy constituents, it follows that $P$ breaks up into $L(\mathfrak{B})$ constituents the degrees of which are the degrees of the Loewy constituents $\mathfrak{R}_{L}(\mathfrak{B}), \cdots, \mathfrak{R}_{1}(\mathfrak{B})$. This holds for every $P$ in $\mathfrak{S}$, and hence for $\mathfrak{S}$.

Clifford's case is obtained by taking for $\mathfrak{B}$ a normal subgroup of an irreducible group $(S)$ of matrices. Here $\mathfrak{S} \supseteq(S)$ and hence $\mathfrak{S}$ is irreducible. Then (6.4A) shows that $L(\mathfrak{B})=1$, i.e., $\mathfrak{B}$ is completely reducible.

If $\mathfrak{A}$ is an irreducible set, we may apply $(6.4 \mathrm{~A})$ to $\mathfrak{B}=\mathfrak{C}(\mathfrak{A})$. Then $\mathfrak{S} \supseteq \mathfrak{A}$, and hence $\mathfrak{S}$ again is irreducible and $L(\mathfrak{B})=1$, i.e., $\mathfrak{S}(\mathfrak{H})$ is completely reducible. If $\mathfrak{S}(\mathfrak{A})$ had two nonsimilar irreducible constituents, then $\mathfrak{S}(\mathfrak{S}(\mathfrak{A})$ ) would be reducible according to $(6.2 \mathrm{C})$, and hence $\mathfrak{A} \subseteq \mathfrak{S}(\mathfrak{E}(\mathfrak{A}))$ would be reducible. This gives

(6.4B) If $\mathfrak{A}$ is an irreducible set of matrices, $\mathfrak{(}(\mathfrak{A})$ is completely reducible, and all its irreducible constituents are similar.

From $(6.2 \mathrm{C})$, we also obtain

(6.4C) If $\mathfrak{A}$ is completely reducible, so is $\mathfrak{\complement}(\mathfrak{A})$.

5. For the actual construction of intertwining matrices, the following remark is sometimes useful.

(6.5A) Let $\mathfrak{A}$ and $\mathfrak{B}$ be related sets of matrices and assume that $\mathfrak{A}$ consists

${ }^{21}$ Cf. Weyl $[31$, p. 86]. The basis of the argument is the following remark. If $\mathfrak{A} \neq\{0\}$ is an irreducible semigroup of matrices of degree $a$, if $Z \neq 0$ is a fixed $a$-dimensional vector, then every $a$-dimensional vector can be written as a finite sum $\sum A Z c_{A}$ where the $A$ are elements of $\mathfrak{X}$ and the $c_{A}$ are elements of $K$. If this were not so, the vectors of this type would form an invariant subspace.

(22) Clifford [6]. 
of nonsingular matrices. For corresponding matrices $A$ and $B$, let the vector $U$ undergo the transformation contragredient to $A$, and let $X$ undergo the transformation $B$; i.e.,

$$
U \rightarrow U^{*}=U A^{-1}, \quad X \rightarrow X^{*}=B X .
$$

The matrix $P$ intertwines $\mathfrak{A}$ and $\mathfrak{B}$, if and only if $U P X$ is an invariant for each pair of corresponding transformations (18).

Indeed, from $U^{*} P X^{*}=U P X$, it follows that $U A^{-1} P B X=U P X$ for all $U$ and $X$, and hence $A^{-1} P B=P$.

6. We conclude this section by proving some properties of the Loewy constituents of reducible sets.

(6.6A) If $\mathfrak{A}$ is a reducible set of matrices

$$
\mathfrak{A} \sim\left(\begin{array}{ll}
\mathfrak{S} & \\
\mathfrak{J} & \mathfrak{S}
\end{array}\right),
$$

then $\mathfrak{R}_{i}(\mathfrak{H})$ splits into $\mathfrak{R}_{i}(\mathfrak{S})$ and constituents of $\mathfrak{R}_{1}(\mathfrak{S}), \mathfrak{R}_{2}(\mathfrak{S}), \cdots, \mathfrak{R}_{i}(\mathfrak{H})\left({ }^{23}\right)$. Similarly, $\tilde{\mathfrak{Q}}_{i}(\mathfrak{H})$ splits into $\tilde{\mathfrak{R}}_{i}(\mathfrak{S})$ and constituents of $\tilde{\mathfrak{R}}_{1}(\mathfrak{S}), \tilde{\mathfrak{R}}_{2}(\mathfrak{S}), \cdots, \tilde{\mathfrak{l}}_{i}(\mathfrak{S})\left({ }^{23}\right)$.

Proof. We may assume that $(\mathfrak{S}$ and $\mathfrak{S}$ both appear in their lower Loewy normal forms. In order to find $\ell_{1}(\mathfrak{U})$, we may use the method of $\S 5.3$. It is obvious that $\mathfrak{R}_{1}(\mathfrak{A})$ will be built up from $\mathfrak{R}_{1}(\mathfrak{L})$ and, perhaps, some constituents of $\mathfrak{R}_{1}(\mathfrak{S})$. We may assume that all these constituents stand in columns which otherwise consist of zeros. Removing the rows and columns of these constituents from $\mathfrak{A}$, we obtain a set

$$
\mathfrak{A}^{*}=\left(\begin{array}{cc}
\mathfrak{S}^{*} & \\
\mathfrak{S}^{*} & \mathfrak{S}^{*}
\end{array}\right)
$$

where $\mathfrak{S}^{*}$ is a top constituent of $\mathfrak{S}$, and $\mathfrak{S}^{*}$ a top constituent of $\mathfrak{S}$. It is easily seen, using the same method, that if an irreducible constituent of $\mathfrak{F}^{*}$ belongs

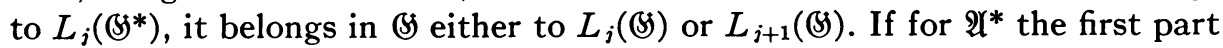
of the statement has been proved, as we may assume, it follows easily for $B$. The second part is obtained from the first by going over to the transposed matrix as in $\$ 5.4$.

As a corollary:

(6.6B) We have $L(\mathfrak{A}) \geqq L(\mathfrak{H})$ and $L(\mathfrak{A}) \geqq L(\mathfrak{S})$.

The situation is far simpler, if $\Im=0$ in (19) of $(6.6 \mathrm{~A})$. We then have the following:

(6.6C) If the set $\mathfrak{A}$ breaks up completely into two constituents $\mathbb{S}$ and $\mathfrak{S}$, then

${ }^{(23)}$ Some of these constituents may be missing. 
$L_{i}(\mathfrak{H})$ breaks up into $L_{i}(\mathfrak{S})$ and $L_{i}(\mathfrak{S}) ; \tilde{L}_{i}(\mathfrak{A})$ breaks up into $\tilde{L}_{i}(\mathfrak{S})$ and $\tilde{L}_{i}(\mathfrak{S})\left({ }^{24}\right)$. Further, $L(\mathfrak{H})=\max (L(\mathfrak{S}), L(\mathfrak{S}))$.

The proof again is obtained by the method of $\$ 5.3$ and is similar to, but simpler than that of $(6.6 \mathrm{~A})$.

\section{GROUP PAIRS AND ASSOCIATED SETS OF MATRICES}

1. Consider three Abelian groups $\mathfrak{U}, \mathfrak{B}$, and $\mathfrak{B}$, each written with addition as group combination. We assume that the "product" $u v$ of an element $u$ of $\mathfrak{U}$ with an element $v$ of $\mathfrak{B}$ is defined as an element of $\mathfrak{B}$ such that the distributive laws hold,

$$
\left(u_{1}+u_{2}\right) v=u_{1} v+u_{2} v, \quad u\left(v_{1}+v_{2}\right)=u v_{1}+u v_{2},
$$

for any $u, u_{1}, u_{2}$ in $\mathfrak{U}$ and any $v, v_{1}, v_{2}$ in $\mathfrak{B}\left({ }^{25}\right)$.

If $\mathfrak{U}$ has a set of operators $\Gamma$, and $\mathfrak{B}$ a set of operators $\Delta$, we write the operation in $\mathfrak{U}$ as $l$-multiplication and the operation in $\mathfrak{B}$ as $r$-multiplication. We then assume that $\mathfrak{W}$ possesses the two sets of operators $\Gamma$ and $\Delta$, the first corresponding to $l$-multiplication and the second to $r$-multiplication, and that the associative laws hold,

$$
\gamma(u v)=(\gamma u) v, \quad(u v) \delta=u(v \delta), \quad \gamma(w \delta)=(\gamma w) \delta,
$$

for any $u$ in $\mathfrak{U}, v$ in $\mathfrak{B}, w$ in $\mathfrak{B}, \gamma$ in $\Gamma, \delta$ in $\Delta$. If all these conditions are satisfied, we say that $(\mathfrak{U}, \mathfrak{B})$ is a group pair.

An $r$-annihilator $v_{0}$ is an element of $\mathfrak{B}$ for which $\mathfrak{U} v_{0}=0$, i.e., $u v_{0}$ is the zeroelement of $\mathfrak{B}$ for every $u$ in $\mathfrak{U}$. All these $r$-annihilators form an (admissible) subgroup $\mathfrak{B}_{0}$ of $\mathfrak{B}$. Similarly, the $l$-annihilators $u_{0}$ in $\mathfrak{U}$ with $u_{0} \mathfrak{B}=0$ form a subgroup $\mathfrak{U}_{0}$ of $\mathfrak{u}$. If we set $\left(\mathfrak{U}_{0}+u\right)\left(\mathfrak{B}_{0}+v\right)=u v$, then $\left(\mathfrak{U} / \mathfrak{U}_{0}, \mathfrak{B} / \mathfrak{B}_{0}\right)$ becomes a group pair in which there are no $l$-annihilators or $r$-annihilators except the zero elements. Such a group pair is said to be primitive $\left({ }^{26}\right)$.

2 . Let $(\mathfrak{U}, \mathfrak{B})$ be a group pair in which the zero element is the only $l$-annihilator: $\mathfrak{U}_{0}=0$. We consider a set $\mathfrak{B}$ of homomorphic $\left({ }^{27}\right)$ mappings $B$ of $\mathfrak{B}$ upon itself or a subgroup of $\mathfrak{B}$. We say that the group pair $(\mathfrak{U}, \mathfrak{B})$ admits the transformations $B$ of $\mathfrak{B}$, if to each $B: v \rightarrow v^{*}$ there corresponds a transformation $A: u \rightarrow u^{*}$ of $\mathfrak{u}$ upon itself or a subgroup of $\mathfrak{u}$, such that

$$
u^{*} v=u v^{*}
$$

for all $u$ in $\mathfrak{U}$ and all $v$ in $\mathfrak{B}$. The element $u^{*}$ is uniquely determined by (20), if $B$ and $u$ are given. Further

\section{(7.2A) The mapping $A$ is a homomorphism.}
(24) Some of these constituents may be missing.
(25) Such group pairs $\mathfrak{u}, \mathfrak{B}$ have first been considered by Pontrjagin [23].
(26) Cf. Pontrjagin [23].
${ }^{(27)}$ As always, this is to mean operator-homomorphic mappings. 
Proof. We have (for $u, u_{1}, u_{2}$ in $\mathfrak{u}, v$ in $\mathfrak{B}, \gamma$ in $\Gamma$ )

$$
\begin{aligned}
\left(u_{1}+u_{2}\right)^{*} v & =\left(u_{1}+u_{2}\right) v^{*}=u_{1} v^{*}+u_{2} v^{*}=u_{1}^{*} v+u_{2}^{*} v=\left(u_{1}^{*}+u_{2}^{*}\right) v \\
(\gamma u)^{*} v & =(\gamma u) v^{*}=\gamma\left(u v^{*}\right)=\gamma\left(u^{*} v\right)=\left(\gamma u^{*}\right) v
\end{aligned}
$$

which imply $\left(u_{1}+u_{2}\right)^{*}=u_{1}^{*}+u_{2}^{*},(\gamma u)^{*}=\gamma u^{*}$.

We call the set $\mathfrak{A}$ of all these transformations $A$ the set which is associated with $\mathfrak{B}$ by the group pair $(\mathfrak{U}, \mathfrak{B})$. Because of the symmetry of $(20)$ we have

(7.2B) If the group pair $(\mathfrak{U}, \mathfrak{B})$ is primitive, the relationship between $\mathfrak{A}$ and $\mathfrak{B}$ is reciprocal.

Indeed, if we start from the mapping $A: u \rightarrow u^{*}$ of $\mathfrak{U}$, we see from (20) that the pair $(\mathfrak{U}, \mathfrak{B})$ admits the transformations of $\mathfrak{A}$, and that $\mathfrak{B}$ is the associated set.

3 . Let $(\mathfrak{U}, \mathfrak{B})$ be again a group pair with 0 as the only $l$-annihilator. Every element $u$ generates a homomorphic mapping $v \rightarrow u v$ of $\mathfrak{B}$ upon a subgroup of $\mathfrak{B}$ which is an operator-homomorphism with regard to the operators of $\Delta$. All such operator-homomorphic mappings of $\mathfrak{B}$ upon a subgroup of $\mathfrak{B}$ form an additive group $\overline{\mathfrak{U}}$ which possesses the elements of $\Gamma$ as $l$-operators. Then $\mathfrak{U}$ is (operator-) isomorphic with a subgroup of $\overline{\mathfrak{u}}$; we may consider $\mathfrak{U}$ itself as a subgroup of $\overline{\mathfrak{u}}$.

If $B: v \rightarrow v^{*}$ is a homomorphic mapping of $\mathfrak{B}$ upon $\mathfrak{B}$ or a subgroup of $\mathfrak{B}$, and if $\bar{u}$ is any element of $\overline{\mathfrak{u}}$, then $v \rightarrow \bar{u} v^{*}$ is an operator-homomorphic mapping of $\mathfrak{B}$ upon a subgroup of $\mathfrak{B}$ (with regard to the operators of $\Delta$ ). It then is given by an element $\bar{u}^{*}$ of $\overline{\mathfrak{u}}$, and we have $\bar{u}^{*} v=\bar{u} v^{*}$. Hence

(7.3A) If $(\mathfrak{U}, \mathfrak{B})$ is a group pair without nonzero l-annihilators, we can replace $\mathfrak{U}$ by a larger group $\overline{\mathfrak{U}}$ such that $(\overline{\mathfrak{U}}, \mathfrak{B})$ admits every set $\mathfrak{B}$ of homomorphic mappings of $\mathfrak{B}$ upon a subgroup of $\mathfrak{B}$.

4. Let us restrict ourselves to the case that $\mathfrak{U}$ is a contragredient vector space and $\mathfrak{B}$ a cogredient vector space, the coordinates of the vectors taken from a fixed division ring $K$. We then take $\Gamma=\Delta=K$ in $\S 7.1$, and assume that $\mathfrak{W}$ is an $m$-dimensional cogredient vector space, and that $l$-multiplication of an element $W$ with an element $\kappa$ of $K$ is performed by $l$-multiplying each component of $W$ with $\kappa\left({ }^{28}\right)$. We say in this case that $(\mathfrak{U}, \mathfrak{B})$ form a group pair of rank $m$. Assume that 0 is the only $l$-annihilator.

Let $n$ be the number of dimensions of $\mathfrak{B}$. Since every element $\bar{U}$ of $\overline{\mathfrak{U}}$ corresponds to an operator-homomorphic mapping of $\mathfrak{B}$ upon a subgroup of $\mathfrak{B}$ (with regard to $r$-operators), it is given by a matrix of type $(m, n)$ with coefficients in $K$. We may identify $\bar{U}$ with this matrix; the products $\kappa \bar{U}$ and $\bar{U} V$

(28) We may then consider $\mathfrak{W}$ also as a contragredient vector space, if we consider only the addition in $\mathfrak{W}$ and the $l$-multiplication with elements of $K$. There will be no danger of a confusion, since we shall not perform linear transformations in $\mathfrak{W}$. 
for $\kappa$ in $K, V$ in $\mathfrak{B}$ then have the ordinary significance (cf. $\$ 3$ ). The number of dimensions of $\overline{\mathfrak{u}}$ is $m n$.

Every mapping $B$ of $\mathfrak{B}$ of the kind considered in $\$ 7.3$ is a linear transformation $V \rightarrow V^{*}$ and hence given by a matrix $\left(b_{\kappa \lambda}\right)$ of degree $n$ which we also denote by $B$ setting $V^{*}=B V$. The associated mapping $\bar{A}: \bar{U} \rightarrow \bar{U}^{*}$ of $\mathfrak{u}$ is defined by $\bar{U}^{*} V=\bar{U} V^{*}$ or $\bar{U}^{*} V=\bar{U} B V$ which implies $\bar{U}^{*}=\bar{U} B$. This, of course, is a linear transformation $\bar{A}$ of $\overline{\mathfrak{u}}$ whose matrix we also denote by $\bar{A}$. We may consider $\overline{\mathfrak{u}}$ as a direct sum of $m n$-dimensional vector spaces $\mathfrak{T}_{1}, \cdots, \mathfrak{T}_{m}$ where in the matrices of $\mathfrak{I}_{i}$ only the coefficients in the $i$ th row are different from 0 . If we choose a basis $E_{j}^{(i)}$ of $\mathfrak{T}_{i}$ by taking the $j$ th coefficients of the $i$ th row equal to 1 , and all the other coefficients equal to 0 , we see that $\bar{A}$ transforms $E_{j}^{(i)}$ into $E_{j}^{(i)} B=\sum b_{j \lambda} E_{\lambda}^{(i)}$. This proves $\mathfrak{T}_{i}$ invariant under $\bar{A}$, the matrix of the induced transformation being $B$. Hence $\bar{A}=m \times B$. The set $\overline{\mathfrak{A}}$ associated with a set $\mathfrak{B}$ of transformations $B$ by the pair $(\mathfrak{U}, \mathfrak{B})$ is then $\overline{\mathfrak{A}}=m \times \mathfrak{B}$.

If $\mathfrak{U}$ is a subgroup of $\overline{\mathfrak{U}}$, and the group pair $(\mathfrak{U}, \mathfrak{B})$ admits the transformations of $\mathfrak{B}$, then $\mathfrak{U}$ must be a subspace of $\overline{\mathfrak{u}}$ invariant under $\overline{\mathfrak{A}}$. The transformations of $\mathfrak{U}$ induced by $\overline{\mathfrak{A}}$ form a top constituent $\mathfrak{A}$ of $\overline{\mathfrak{A}}$, and this $\mathfrak{A}$ is the set associated with $\mathfrak{B}$ by the group pair $(\mathfrak{U}, \mathfrak{B})$. Hence (cf. $\S 4.3$ )

(7.4A) Let $\mathfrak{U}$ be a contragredient vector space and $\mathfrak{B}$ a cogredient vector space both forming a group pair of rank $m$. If 0 is the only l-annihilator, and $(\mathfrak{U}, \mathfrak{B})$ admits the set $\mathfrak{B}$ of homomorphic mappings of $\mathfrak{B}$ upon $\mathfrak{B}$ or a subgroup of $\mathfrak{B}$, then the associated set $\mathfrak{A}$ is a top constituent of $m \times \mathfrak{B}$.

In the same manner, we prove

(7.4B) If 0 is the only r-annihilator in $(\mathfrak{U}, \mathfrak{B})$, and $(\mathfrak{U}, \mathfrak{B})$ admits the set $\mathfrak{A}$ of homomorphic mappings of $\mathfrak{U}$ upon a subgroup of $\mathfrak{U}$, then the associated set of transformations of $\mathfrak{B}$ is an end constituent of $m \times \mathfrak{A}$.

That we here obtain an end constituent instead of a top constituent as in (7.4A) is due to the fact that $\mathfrak{B}$ is a cogredient vector space. The transformations induced in an invariant subspace are end constituents (cf. §4.3).

5 . Let us apply the preceding considerations to sets $\mathfrak{B}$ of matrices of degree $n$ with coefficients in the division ring $K$. Let $m>0$ be a given integer. We say that a set $\mathfrak{U}$ of matrices of type $(m, n)$ with coefficients in $K$ is a $(K, \mathfrak{B})$-double module, if $\mathfrak{U}$ contains the matrices $U_{1}+U_{2}, \kappa U, U B$ for any $U, U_{1}, U_{2}$ in $\mathfrak{U}$, any $\kappa$ in $K$, and any $B$ in $\mathfrak{B}$. We then choose an $l$-basis $U_{1}, U_{2}, \cdots, U_{k}$ of $\mathfrak{u}$. Since any product $U_{\kappa} B$ lies in $\mathfrak{U}$ again, we have formulae

$$
U_{\kappa} B=\sum_{\lambda=1}^{k} a_{\kappa \lambda} U_{\lambda}, \quad \kappa=1,2, \cdots, k,
$$

with coefficients $a_{\kappa \lambda}$ in $K$. We say that the set $\mathfrak{A}$ of all the matrices $A=\left(a_{\kappa \lambda}\right)$ is the set associated with $\mathfrak{B}$ by the double module $\mathfrak{U}$. The degree $k$ of $\mathfrak{A}$ is the 
$l$-rank of $\mathfrak{U}$. If $\mathfrak{B}$ is closed under addition or multiplication, the set $\mathfrak{A}$ is homomorphic with $\mathfrak{B}$ with regard to this operation $\left({ }^{29}\right)$. If the $l$-basis $U_{k}$ is replaced by another $l$-basis, $\mathfrak{A}$ is replaced by a similar set.

If $\mathfrak{B}$ is the $n$-dimensional cogredient vector space in which the transformations of $\mathfrak{B}$ take place, then $(\mathfrak{U}, \mathfrak{B})$ form a group pair, the product $U V$ of a matrix $U$ of $\mathfrak{U}$ and a vector $\mathfrak{B}$ being defined in the ordinary manner. This group pair $(\mathfrak{U}, \mathfrak{B})$ is of rank $m$, and 0 is the only $l$-annihilator.

Further, $(\mathfrak{U}, \mathfrak{B})$ admits the transformations $\mathfrak{B}$ of $\mathfrak{B}$, and $\mathfrak{A}$ is the associated set in the sense of $\$ 7.2$, since the transformation $U_{\kappa} \rightarrow U_{\kappa} B$ in the contragedient vectors space with the basis $U_{1}, U_{2}, \cdots, U_{k}$ has the matrix $A$ according to (21). From (7.4A) there follows

(7.5A) If $\mathfrak{U}$ is a $(K, \mathfrak{B})$-double module, consisting of matrices of type $(m, n)$, then $\mathfrak{U}$ associates the set of matrices $\mathfrak{B}$ of degree $n$ with a set $\mathfrak{A}$ which is a top constituent of $m \times \mathfrak{B}$.

The $r$-annihilators of $(\mathfrak{U}, \mathfrak{B})$ will form a subspace $\mathfrak{B}_{0}$ of $\mathfrak{B}$ which is invariant under $\mathfrak{B}$ since $\mathfrak{U} \cdot B V_{0} \subseteq \mathfrak{U} V_{0}=(0)$ for $V_{0}$ in $\mathfrak{B}_{0}, B$ in $\mathfrak{B}$. Let $\mathfrak{B}_{0}$ be the set of transformations of $\mathfrak{B} / \mathfrak{B}_{0}$ induced by $\mathfrak{B}$; then $\mathfrak{B}_{0}$ is a top constituent of $\mathfrak{B}$ according to $\S 4.3$. We may consider $\left(\mathfrak{U}, \mathfrak{B} / \mathfrak{V}_{0}\right)$ as a primitive group pair consisting of a contragredient vector space $\mathfrak{U}$ and a cogredient vector space $\mathfrak{B} / \mathfrak{B}_{0}$. The rank of this group pair still is $m$. If $B: V \rightarrow V^{*}$ is a transformation of $\mathfrak{B}$, and $A: U \rightarrow U^{*}$ the corresponding transformation of $\mathfrak{A}$, then we have $U^{*} V=U V^{*}$. The corresponding equation holds, when we replace $V$ and $V^{*}$ by their residue class modulo $\mathfrak{B}_{0}$. Then $V^{*} \equiv B_{0} V\left(\bmod \mathfrak{B}_{0}\right)$ where $B_{0}$ is the matrix of $\mathfrak{B}_{0}$ corresponding to $B$ in $\mathfrak{B}$. Consequently, the group pair $\left(\mathfrak{U}, \mathfrak{B} / \mathfrak{B}_{0}\right)$ associates the set of transformations $\mathfrak{B}_{0}$ of $\mathfrak{B} / \mathfrak{B}_{0}$ with the set $\mathfrak{A}$ of transformations of $\mathfrak{U}$ and vice versa (cf. (7.2B)).

Then from $(7.4 \mathrm{~B})$ we obtain

(7.5B) In (7.5A) let $\mathfrak{B}_{0}$ be the set of all $n$-dimensional vectors $V_{0}$ for which $U V_{0}=0$ for every $U$ in $\mathfrak{U}$. Then $\mathfrak{B}_{0}$ is invariant under $\mathfrak{B}$. If $\mathfrak{B}_{0}$ is the top constituent of $\mathfrak{B}$, consisting of the transformations of $\mathfrak{B} / \mathfrak{B}_{0}$ induced by $\mathfrak{B}$, then $\mathfrak{B}_{0}$ is an end constituent of $m \times \mathfrak{A}$.

6. We can now apply (6.6A), (6.6B), and (6.6C) and obtain

(7.6A) In the notation of $(7.5 \mathrm{~A})$ and $(7.5 \mathrm{~B}) \mathfrak{A}$ and $\mathfrak{B}_{0}$ have the same number of Loewy constituents: $L(\mathfrak{H})=L\left(\mathfrak{B}_{0}\right)$. Every irreducible constituent of $\mathfrak{R}_{i}\left(\mathfrak{B}_{0}\right)$ appears in $\mathfrak{R}_{i}(\mathfrak{H})$, and every irred ucible constituent of $\mathfrak{R}_{i}(\mathfrak{H})$ appears in some $\mathfrak{R}_{i+j}\left(\mathfrak{B}_{0}\right)$ with $j \geqq 0$. Every irreducible constituent $\widetilde{\mathfrak{l}}_{i}(\mathfrak{H})$ appears in $\tilde{\mathfrak{R}}_{i}\left(\mathfrak{B}_{0}\right)$, and every irreducible constituent of $\tilde{\mathfrak{R}}_{i}\left(\mathfrak{B}_{0}\right)$ appears in some $\tilde{\mathfrak{R}}_{i+j}(\mathfrak{H})$ with $j \geqq 0$.

We have the corollary

${ }^{(29)}$ In the notation of $E$. Noether [20], $\mathfrak{U}$ is a representation module for the representation $\mathfrak{A}$ of $\mathfrak{B}$. 
(7.6B) The sets $\mathfrak{A}$ and $\mathfrak{B}_{0}$ have the same irreducible constituents though not necessarily with the same multiplicities.

It is also possible to make some statements concerning the multiplicities, e.g.,

(7.6C) If an irreducible constituent $\mathfrak{F}$ appears $h$ times in $L_{i}\left(\mathfrak{B}_{0}\right)$, it appears at least $h / m$ times in $L_{i}(\mathfrak{H})$. (Similarly in the other cases.)

7. As an application, we prove the following theorem:

(7.7A) Let $\mathfrak{B}$ be a set of matrices which has no constituents $(0)$, and let $\mathfrak{u}$ be a $(K, \mathfrak{B})$ double module consisting of matrices of type $(m, n)$. The necessary and sufficient condition that a matrix $Z$ of type $(m, n)$ belongs to $\mathfrak{U}$ is that $Z B$ belongs to $\mathfrak{U}$ for every $B$ in $\mathfrak{B}$.

Proof. Let $U_{1}, U_{2}, \cdots, U_{k}$ be an $l$-basis of $\mathfrak{u}$. If $Z$ does not belong to $\mathfrak{u}$, then $U_{1}, U_{2}, \cdots, U_{k}, Z$ will be an $l$-basis of a $(K, \mathfrak{B})$-double module $\mathfrak{U}^{*}$. The set associated with $\mathfrak{B}$ by $\mathfrak{U}^{*}$ has the form

$$
\mathfrak{A}^{*}=\left(\begin{array}{cc}
\mathfrak{A} & \\
* & 0
\end{array}\right)
$$

where $\mathfrak{A}$ is the associated set with $\mathfrak{B}$ by $\mathfrak{U}$. According to (7.6A) every irreducible constituent of $\mathfrak{A}^{*}$ must appear in $\mathfrak{B}$ whereas 0 is no constituent of $\mathfrak{B}$. Hence $Z$ must belong to $\mathfrak{U}$.

8. Finally, we give some formulae showing the relationship between $\mathfrak{A}$ and $\mathfrak{B}$ in a more formal manner.

(7.8A) Let $\mathfrak{A}$ and $\mathfrak{B}$ be two related sets of matrices of degrees $k$ and $n$ respectively, let $m$ be a positive integer, and $h_{\mu \nu}^{(\kappa)}$ a set of $k m n$ elements of $K$ $(\kappa=1,2, \cdots, k ; \mu=1,2, \cdots, m ; \nu=1,2, \cdots, n)$. We form three sets of matrices, $U_{\kappa}$ of type $(m, n), T_{\nu}$ of type $(k, m)$ and $P_{\mu}$ of type $(k, n)$ :

$$
U_{\kappa}=\left(h_{\alpha \beta}^{(\alpha)}\right) ; \quad T_{\nu}=\left(h_{\beta \nu}^{(\alpha)}\right) ; \quad P_{\mu}=\left(h_{\mu \beta}^{(\alpha)}\right),
$$

where $\alpha$ is the row index and $\beta$ the column index. The three sets of relations (for corresponding $A=\left(a_{\alpha \beta}\right)$ and $\left.B=\left(b_{\alpha \beta}\right)\right)$

$$
\begin{aligned}
U_{\alpha} B & =\sum a_{\alpha \beta} U_{\beta}, \\
A T_{\nu} & =\sum T_{\lambda} b_{\lambda \nu}, \\
\mathfrak{A P} P_{\mu} & =P_{\mu} \mathfrak{B}
\end{aligned}
$$

are equivalent.

Proof. All three relations are equivalent to

$$
\sum_{\lambda} h_{\mu \lambda}^{(\alpha)} b_{\lambda \nu}=\sum_{\beta} a_{\alpha \beta} h_{\mu \nu}^{(\beta)}
$$


The equation (23a) is identical with (21). The equation (23b) shows that all matrices of the form $\sum T_{\nu} c_{\nu}, c_{\nu}$ in $K$, form what we may call an ( $\mathfrak{A}, K$ ) double module $\mathfrak{T}$. If the $T_{\nu}$ are $r$-independent, this $\mathfrak{T}$ associates $\mathfrak{A}$ with $\mathfrak{B}$, and this again expresses the reciprocity between $\mathfrak{B}$ and $\mathfrak{A}$.

\section{The REgUlar REPRESENTATION}

1. We now consider a set (B) of square matrices which forms a semi-group, i.e., which contains the product of any two of its matrices. Let $U_{1}, U_{2}, \cdots, U_{k}$ be an $l$-basis of $\$ S$. The linear combinations $\sum c_{k} U_{k}$ with arbitrary coefficients in $K$ form a $(K,(3)$-double module which we call the enveloping module $\mathfrak{M}(\mathbb{B})$ of $(5)$. For $G$ in $(\$)$, we have the formulae

$$
U_{k} G=\sum_{\lambda} r_{k \lambda} U_{\lambda}, \quad \quad r_{k \lambda} \text { in } K,
$$

and the matrices $R=\left(r_{k \lambda}\right)$ form the associated set $\Re$. The mapping $G \rightarrow R$ is a homomorphism with regard to multiplication. In other words, $\Re$ is a repre-

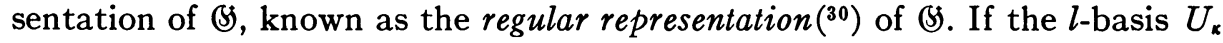
is replaced by another $l$-basis of $\mathfrak{M}\left((\mathfrak{B})\right.$, then $\Re$ is replaced by a similar set $\left({ }^{31}\right)$. The degree of the regular representation is equal to the $l$-rank of $B$.

2 . Let $\mathfrak{B}$ be the space in which the transformations of $\mathbb{B}$ take place. We shall apply (7.5A) and (7.5B) (for $\mathfrak{U}=\mathfrak{M}(\mathfrak{S})$ ). Here $\mathfrak{B}_{0}$ consists of those vectors $V$ for which $\mathfrak{M}(\$) V=0$. This condition is equivalent with $\$ V=0$, and hence $B$ induces the transformation 0 in $\mathfrak{B}_{0}$. It follows that in a suitable coordinate system

$$
\text { (B) } \sim\left(\begin{array}{cc}
\mathfrak{S}_{0} & \\
* & 0
\end{array}\right),
$$

where the constituent 0 at the bottom is of degree $n_{0} \geqq 0\left({ }^{32}\right)$. It is not possible to find a similar set with a bottom constituent 0 of higher degree. From the theorems in $\$ 7.5$ and $\$ 7.6$ we derive:

(8.2A) Let (\$s be a semigroup of matrices of degree $n$. We split \& into a constituent $\mathrm{B}_{0}$ and a bottom constituent 0 of highest possible degree, (25). The regular representation $\Re$ of $\mathfrak{B}$ is a top constituent of $n \times \mathrm{S}_{0}$, and $\mathrm{BH}_{0}$ is an end constituent of $n \times \Re$.

(8.2B) We have $L(\Re)=L\left(\$_{0}\right)$. Every irreducible constituent of $L_{i}\left(\$_{0}\right)$ appears in $\mathfrak{R}_{i}(\Re)$, and every irreducible constituent of $\mathfrak{R}_{i}(\Re)$ appears in some $\mathfrak{R}_{i+j}\left(\mathbb{S}_{0}\right)$

$\left.{ }^{(30}\right)$ For properties of the regular representation, cf. Frobenius [9], MacDuffee [16], Brauer and Nesbitt [4], Nesbitt [19], Nakayama [18].

${ }^{(31)}$ It should be noticed that in the case of a non-commutative $K$, the module $\mathfrak{M}(\mathbb{S})$ is, in general, not a ring. Further, similar semi-groups $B$ and $\mathcal{S}_{1}$ may have different $l$-ranks and different regular representations.

(32) If $n_{0}=0$, then the constituent 0 in (25) is missing. 
with $j \geqq 0$. Every irreducible constituent of $\tilde{\mathfrak{R}}_{i}(\Re)$ appears in $\tilde{\mathfrak{R}}_{i}\left(\mathfrak{S}_{0}\right)$, and every irreducible constituent of $\tilde{\mathfrak{R}}_{i}\left(\mathfrak{S}_{0}\right)$ appears in some $\tilde{\mathfrak{R}}_{i+j}(\Re)$ with $j \geqq 0$.

These results lead to the following corollaries:

(8.2C) We have either $L(\Re)=L(\mathfrak{S})$, or $L(\Re)=L(\$)-1$. If $\mathfrak{R}_{1}(\mathfrak{S})$ does not contain a constituent 0 , we have the first case.

(8.2D) The sets $\&$ and $\Re$ have the same irreducible constituents, except perhaps constituents 0 which may appear in (5) without appearing in $\Re$.

(8.2E) If $\mathbb{B}$ is completely reducible, so is $\Re$.

For $L(\$)=1$ implies $L(\Re)=1$ by $(8.2 \mathrm{C})$, and this is equivalent to the complete reducibility of $\Re$.

In certain cases, $\mathfrak{S}_{0}$ can be replaced by $\mathfrak{B}$. We can prove

(8.2F) If $\mathfrak{M}(\$)$ contains a matrix $J \neq 0$ such that $J G=G$ for every $G$ in $(\$)$, then (S) splits completely into $\mathfrak{S}_{0}$ and a constituent 0 , and we have $L(\Re)=L(\mathbb{B})$ $=L\left(\$_{0}\right), \mathfrak{R}_{i}(\$)=\mathfrak{R}_{i}\left(\$_{0}\right)$ for every $i \geqq 2$. The assumption is satisfied, in particular, when (S) has a l-unit $J$.

Proof. Assume that $Q^{-1}\left(S Q\right.$ splits in the form (25). The last $n_{0}$ columns in all the matrices of $Q^{-1} \mathbb{S} Q$ vanish. The same then is true for $Q \cdot Q^{-1} \mathbb{S} Q=\mathbb{S} Q$,

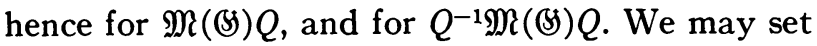

$$
Q^{-1} \mathscr{S}=\left(\begin{array}{cc}
\Im_{0} & \\
\Im & 0
\end{array}\right), \quad Q^{-1} J Q=\left(\begin{array}{ll}
X & 0 \\
Y & 0
\end{array}\right)
$$

since $J$ belongs to $\mathfrak{M}(\mathfrak{S})$. From $J G=G$, we obtain $Y \mathbb{S}_{0}=\mathbb{C}$ or $\mathbb{S}=Y \mathbb{S}_{0}-0 Y$. This shows that after an elementary similarity transformation, we may replace $(5$ by 0 . This shows the first part of $(8.2 \mathrm{~F})$; the other statements follow from it.

From (7.7A), we obtain at once

(8.2G) Let (\$) be a semigroup of matrices of degree $n$ which has no constituent 0 . A necessary and sufficient condition that a matrix $Z$ of degree $n$ belongs to $\mathfrak{M}(\mathfrak{S})$ is that $Z G$ belongs to $\mathfrak{M}(\mathbb{S})$ for every $G$ in $\mathfrak{B}$. In particular, the unit matrix I belongs to $\mathfrak{M}(\mathfrak{M})$.

3. In certain cases, the theorem (8.2B) can be improved. We prove:

(8.3A) Assume that the semigroup \& itself appears in its lower Loewy normal form, and that no constituent 0 appears in $(S)$. Every irreducible constituent of $\mathfrak{R}_{i}(\mathfrak{S})$ is also a constituent of $\mathfrak{R}_{1}(\Re), L_{2}(\Re), \cdots, \mathfrak{R}_{i}(\Re)$.

Proof. Assume that the semigroup $(S$ itself splits into several constituents, one of which is $\mathfrak{S}$. Denote by $W_{1}, W_{2}, \cdots, W_{k}$ the matrices of $\mathfrak{M}(\mathfrak{S})$ which correspond to an $l$-basis $U_{1}, U_{2}, \cdots, U_{k}$ of $\mathfrak{M}(\mathfrak{S})$. Obviously, we can choose 
$U_{1}, U_{2}, \cdots, U_{k}$ such that $W_{1}=\cdots=W_{j}=0$ and $W_{j+1}, \cdots, W_{k}$ form an $l$-basis of $\mathfrak{M}(\mathfrak{S})$. If $G$ in $(S)$ corresponds to $H$ in $\mathfrak{S}$, then (24) implies

$$
W_{\kappa} H=\sum r_{\kappa \lambda} W_{\lambda}
$$

and we easily see that $\Re$ splits into a top constituent of degree $j$ and the regular representation $\Re^{*}$ of $\mathfrak{S}$ as end constituent. From (6.6A) and (8.2B) it follows that every irreducible constituent of $\mathfrak{R}_{\nu}(\mathfrak{S})$ appears in $\mathfrak{R}_{\nu}(\Re)$; $(0)$ is not a constituent of $\mathfrak{S}$.

We now choose $\mathfrak{S}$ as the constituent of $\mathbb{S}$ which contains the Loewy constituents $\mathfrak{R}_{L}(\mathfrak{S}), \cdots, \mathfrak{R}_{\beta}(\mathfrak{S})$. Then $\mathfrak{R}_{\nu}(\mathfrak{S})=\mathfrak{R}_{\beta+\nu-1}(\mathfrak{S})$, and for $i=\nu+\beta-1$, $1 \leqq \nu \leqq i$, we obtain the statement of $(8.3 \mathrm{~A})$.

If the underlying division ring is a field, there is no restriction in the assumption that $(\$)$ itself is in its lower Loewy normal form, since similar semigroups here have the same regular representation.

4. A discussion, analogous to that in $\$ 8.1$, is possible with regard to an $r$-basis $\tilde{U}_{1}, \cdots, \tilde{U}_{l}$ of $\$$. Here we set

$$
G \tilde{U}_{\lambda}=\sum_{\kappa} \tilde{U}_{\kappa} s_{k \lambda}, \quad s_{k \lambda} \text { in } K,
$$

and $G \rightarrow S=\left(s_{\kappa \lambda}\right)$ defines the second regular representation of $\$(5)$ Going over to transposed matrices (cf. (3.5)) in (26), we obtain

(8.4A) The second regular representation $\subseteq$ of a semigroup (5) is the transpose of the first regular representation of the transposed set $\left(\mathrm{S}^{\prime}\right.$.

This remark allows us to restrict ourselves to the consideration of the first regular representation.

\section{IRREDUCIBLE SEMIGROUPS}

1. We now consider irreducible semigroups $B \neq(0)$ consisting of square matrices of degree $n$ with coefficients in the division ring $K$. Since the degree of the regular representation equals the $l$-rank of $(B)$, we obtain from (8.2B):

(9.1A) If (S) is an irreducible semigroup of degree $n$ and l-rank $k$, then the regular representation $\Re$ of $\&$ is similar to $(k / n) \times(\$)$. In particular, the l-rank is a multiple of the degree.

We wish to characterize the number $k / n$ by means of the commuting ring $\mathfrak{S}(\$)$ of $(\mathfrak{s}$. Denoting the row $(0, \cdots, 0,1,0, \cdots, 0)$ with the $i$ th component 1 by $E_{i}$, we see that $E_{i} C$ is the $i$ th row of the matrix $C$. We determine the largest number $h$ of indices $\mu_{1}, \mu_{2}, \cdots, \mu_{h}$, with $1 \leqq \mu_{i} \leqq n$, such that conditions

$$
\sum_{\mu} E_{\mu} C_{\mu}=0, \quad C_{\mu} \text { in }\left(\mathfrak{S}(\mathfrak{S}), \mu \text { ranging over } \mu_{1}, \cdots, \mu_{h},\right.
$$

imply $C_{\mu_{i}}=0$ for all $\mu_{i}$. Since all the $C_{\mu} \neq 0$ in $\mathfrak{S}(\mathfrak{S})$ are nonsingular (cf. 
(6.2A)), we have $h \geqq 1$. The $\mu_{i}$ are all distinct, since if for example $\mu_{1}=\mu_{2}$, we could set $C_{\mu_{1}}=-C_{\mu_{2}} \neq 0$, and all the later $C_{\mu}=0$ in (27). We denote this number $h$ as the $h$-number of $\mathfrak{S}(\$)$, and state

(9.1B) The quotient $k / n$ in (9.1A) is equal to the h-number of (5)(\$)).

Proof. Assume first that $h<n$. For any fixed $i=1,2, \cdots, n$, we can find matrices $C_{\mu i}\left(\mu=\mu_{1}, \cdots, \mu_{h}\right)$ and $C_{i}$ in $\mathbb{S}(\$)$ such that

$$
\sum_{\mu} E_{\mu} C_{\mu i}+E_{i} C_{i}=0
$$

and not all $C_{\mu i}, C_{i}$ vanish. Then $C_{i} \neq 0$, because otherwise (28) would be identical with (27) for $C_{\mu i}=C_{\mu}$, and all these matrices would also vanish. Because of (6.2A), $C_{i}$ is nonsingular, and if we $r$-multiply (28) by its reciprocal, we see that we may assume $C_{i}=I$. We then multiply (28) by an arbitrary element $G$ of $(S$, and obtain

$$
0=\sum_{\mu} E_{\mu} C_{\mu i} G+E_{i} G=\sum_{\mu} E_{\mu} G C_{\mu i}+E_{i} G .
$$

Denote by $t_{1}, t_{2}, \cdots, t_{h n}$ the $h n$ coefficients appearing in the rows $\mu_{1}, \mu_{2}, \cdots, \mu_{h}$ of $G$. Since $E_{i} G$ is the $i$ th row of $G$, and $E_{\mu} G$ the $\mu$ th row of $G$, we see from (29) that every fixed coefficient of $G$, say in the $i$ th row and $j$ th column, is a linear function $\sum t_{\rho} \gamma_{\rho}$, where the $\gamma_{\rho}$ are elements of $K$ which are independent of $G$ (but dependent on $i, j$ ). Then $G$ has the form $G=\sum t_{\rho} Q_{\rho}$, where the $Q_{\rho}$ are fixed matrices, and this shows that the $l$-rank $k$ of $\&$ is not larger than $h n$. This is also true, if $h=n$, since certainly $k \leqq n^{2}$. Thus we always have $k / n \leqq h$.

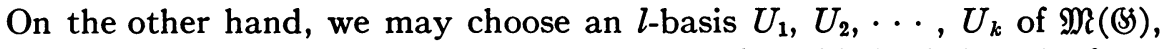
such that the regular representation $\Re$ with regard to this basis has the form (cf. (9.1A))

$$
\Re=j \times \mathfrak{S}, \quad j=k / n .
$$

We now apply (7.8A) to $\mathfrak{A}=\Re$ and $\mathfrak{B}=\mathfrak{B}$, using for $U_{k}$ the notation of the first formula (22) and defining $P_{\mu}$ by the last formula (22); we have here $m=n$. For the $n$ matrices $P_{\mu}$ which intertwine $\Re$ and $\$(\mu=1,2, \cdots, n)$, according to (22), we have

$$
E_{\nu} P_{\mu}=E_{\mu} U_{\nu}=\left(h_{\mu 1}^{(\nu)}, h_{\mu 2}^{(\nu)}, \cdots, h_{\mu n}^{(\nu)}\right) .
$$

We break up each matrix $P_{\mu}$ according to the scheme $(n, n, \cdots, n \mid n)$,

$$
P_{\mu}=\left(\begin{array}{c}
Q_{\mu 1} \\
\vdots \\
\dot{Q}_{\mu j}
\end{array}\right) \text {. }
$$

Because of (30), each $Q_{\mu \rho}$ intertwines $\$$ with $\$$; i.e., $Q_{\mu \rho}$ belongs to $\mathbb{S}(\mathbb{S})$. 
Choose any $j+1$ values $\mu$ from $1,2, \cdots, n$, and consider the $j$ linear equations

$$
\sum Q_{\mu 1} X_{\mu}=0, \cdots, \sum Q_{\mu j} X_{\mu}=0 .
$$

Since the coefficients lie in the division ring $\mathbb{S}(\$)$, and we have more unknowns $X_{i}$ than equations, there is a non-trivial solution $X_{i}$ in $\mathfrak{E}(\mathbb{S})$ ) (cf. (3.4)). Then $\sum P_{\mu} X_{\mu}=0$. On $l$-multiplying by $E_{\nu}$ and using (31), we obtain

$$
\sum_{\mu} E_{\mu} U_{\nu} X_{\mu}=0 \text {. }
$$

We now determine $z_{1}, \cdots, z_{n}$ in $K$ such that $\sum z_{\nu} U_{\nu}=I$. This is possible (cf. (8.2G)). Since $z_{\nu} E_{\mu}=E_{\mu} z_{\nu}, l$-multiplication of (33) with $z_{\nu}$ and addition over $\nu$ yields $\sum E_{\mu} X_{\mu}=0$. Since the $X_{\mu}$ are elements of $\mathfrak{E}(\$)$ which do not all vanish, this is a relation (27). For any $j+1$ indices $\mu$, we have a non-trivial relation of this kind. Hence $j+1>h$, i.e., $j \geqq h$. Because of (30), we have $k / n \geqq h$. Since we also showed $k / n \leqq h$, the statement is proved.

In the notation of the first part of this proof, it now follows that the matrices $Q_{\rho}$ are $l$-independent and belong to $\mathfrak{M}(\mathbb{B})$, since otherwise $\mathfrak{M}(\mathbb{B})$ would have an $l$-rank smaller than $h n$. Further, $t_{1}, \cdots, t_{h n}$ are the coefficients in the rows $\mu_{1}, \cdots, \mu_{h}$ of $\sum t_{\rho} Q_{p}$. Hence

(9.1C) In the notation of (9.1B), the coefficients in $h$ suitable rows $\mu_{1}, \cdots, \mu_{h}$ of a matrix $M$ of $\mathfrak{M ( B ) ~ c a n ~ b e ~ a s s i g n e d ~ a s ~ a r b i t r a r y ~ e l e m e n t s ~ o f ~} K$, and then $M$ is determined uniquely. We can choose the indices $\mu$ as in (27).

2. Let $v$ be the $r$-rank of $\subseteq(\$)$. There exist at most $j v$ matrices (32) which are $r$-independent, since $Q_{\mu \rho}$ lies in $\mathfrak{E}(\mathfrak{S})$, where $j=k / n=h$. If we now choose more than $h v$ distinct indices $\mu$ from $1,2, \cdots, n$ (assuming that $n>h v$ ), then the matrices $P_{\mu}$ are $r$-dependent and we have equations $\sum P_{\mu} x_{\mu}=0\left(x_{\mu}\right.$ in $K$, not all of them 0$)$. We proceed as in the second part of the proof of (9.1B). On $l$-multiplying with $E_{\nu}$ and using (31), we find $\sum E_{\mu} U_{\nu} k_{\mu}=0$ (summed over $\mu$ ). Again, $l$-multiplying by the same $z_{\nu}$ as above and adding, we find $\sum E_{\mu} x_{\mu}=0$. But this implies $x_{\mu}=0$, which gives a contradiction. Hence $n \leqq h v$, which gives

(9.2A) Let $\$$ be an irreducible semigroup of degree $n$. If \&5 has the l-rank $k$, and $\mathfrak{S}(\mathbb{S})$ has the r-rank $v$, then $n^{2} \leqq k v$.

This can be considered as a generalization of Burnside's theorem (cf. §9.4).

3. Consider a similarity transformation applied to the irreducible semigroup (\$). The same transformation, then, is to be applied to $\mathbb{E}(\mathbb{S})$. According to $(6.4 \mathrm{~B})$, the set $\mathfrak{S}(\mathfrak{B})$ has only one irreducible constituent $\mathfrak{W}$, and after the similarity transformation, we may assume that

$$
\mathfrak{s}(\mathfrak{B})=s \times \mathfrak{W}
$$

where $n / s=t$ is the degree of $\mathfrak{W}$. 
We set $\mathfrak{C}(\mathfrak{B})=\mathfrak{T}$. Since $\mathfrak{W}$ is irreducible, $\mathfrak{T}$ is a division ring. From (6.2B)

$$
\mathfrak{S}(\mathfrak{S})=\mathfrak{S}(s \times \mathfrak{B})=[\mathfrak{S}(\mathfrak{W})]_{s}=[\mathfrak{T}]_{s}
$$

and since $\leftrightarrow \subseteq \subseteq((\$)$, we have

$$
\text { (B) } \subseteq[\mathfrak{T}]_{8} \text {. }
$$

The irreducibility of $(S)$ implies the irreducibility of $\mathfrak{T}$, from the first part of theorem (6.3A). Obviously, $\mathfrak{S}(\mathfrak{T}) \supseteq \mathfrak{B}$. If we had $\mathfrak{S}(\mathfrak{T}) \supset \mathfrak{W}$, then, according to $(6.2 \mathrm{~B})$ we would have $\mathfrak{S}(\mathfrak{S}) \supseteq \mathfrak{S}\left([\mathfrak{T}]_{\mathbb{8}}\right)=s \times \mathfrak{S}(\mathfrak{T}) \supset s \times \mathfrak{W}=\mathfrak{S}(\mathfrak{S})$, which is impossible. Hence $\mathfrak{W}$ and $\mathfrak{I}$ both are irreducible division rings consisting of matrices of degree $t$, and each is the commuting set of the other.

We now apply theorem (9.1B) to $\mathfrak{T}$ instead of $(5)$. If $h_{0}$ is the $h$-number of $\mathfrak{S}(\mathfrak{T})=\mathfrak{W}$, and $z$ the $l$-rank of $\mathfrak{T}$, then $h_{0}=z / t$. But (34) shows that the $h$-number of $\mathfrak{S}(\mathbb{S})$ is $h=s h_{0}$, and hence

$$
k / n=h=s h_{0}=s z / t
$$

which implies $k=s^{2} z$ since $n=s t$. Consequently, (S) and $[\mathfrak{T}]_{8}$ have the same $l$-rank, and therefore $\mathfrak{M}(\mathfrak{S})=\mathfrak{M}\left([\mathfrak{T}]_{s}\right)$. Thus we have

(9.3A) Any irreducible semigroup \& of degree $n$ is, after a similarity transformation, contained in a set $[\mathfrak{T}]_{s}$ where $\mathfrak{T}$ is an irreducible set of matrices of degree $n / s=t$ forming a division ring, and (S) and $[\mathfrak{T}]_{s}$ have the same l-rank and hence the same enveloping module, $\mathfrak{M}(\mathfrak{S})=\mathfrak{M}\left([\mathfrak{T}]_{s}\right)$. Further, $\mathfrak{B}=\mathfrak{S}(\mathfrak{T})$ is the only irreducible constituent of $\mathfrak{S}(\mathfrak{S})$ and its multiplicity is $s$, i.e., $\mathfrak{S}(\mathfrak{S})=s \times \mathfrak{W}$. Conversely, $\mathfrak{T}=\mathfrak{S}(\mathfrak{W})$.

Let $v$ be the $l$-rank of $\mathfrak{S}(\mathfrak{S})$ which by $(34)$ is also the $l$-rank of $\mathfrak{B}$, and let $z$ be the $l$-rank of $\mathfrak{T}$. From (35), we obtain

$$
\frac{k v}{n^{2}}=\frac{s z}{t} \frac{v}{n}=\frac{z}{t} \frac{v}{t} .
$$

Both fractions on the right side are integers; they give the multiplicity of $\mathfrak{T}$ and of $\mathfrak{B}$ in their regular representations. The same is true if we take for $v$ the $r$-rank of $\mathfrak{S}(\mathfrak{B})$. Then $v / t$ is the multiplicity of $\mathfrak{B}$ in its second regular representation. Hence we have

(9.3B) If in (9.3A) the set $\mathfrak{T}$ has the l-rank $z$, if $\mathfrak{S}$ has the l-rank $k$, and $\mathfrak{W}$ the l-rank $v$ (or the $r$-rank $v$ ), then $k v / n^{2}=(z / t)(v / t)$ where $z / t$ and $v / t$ are integers.

This gives, of course, the inequality of (9.2A); but it is not sufficient for a proof of $(9.2 \mathrm{~A})$ in the general case, since we applied here a similarity transformation which may have changed the original ranks.

4. If the underlying division ring $K$ is a field $\left({ }^{33}\right)$, then $l$-rank and $r$-rank

${ }^{(33)}$ For this case, compare, for instance, Weyl [31]. 
always coincide. Further $\mathfrak{M}(\mathfrak{T})=\mathfrak{T}$, since every linear combination of elements of $\mathfrak{I}$ commutes with every element $\mathfrak{W}$. Similarly, $\mathfrak{M}(\mathfrak{W})=\mathfrak{W}$.

If $B$ is an irreducible algebra of matrices, then $\mathfrak{M}(\mathfrak{S})=(B)$, and (9.3A) shows that $\mathfrak{B} \sim[\mathfrak{T}]_{s}$ where $\mathfrak{T}$ itself is an irreducible division algebra over $K$. This is Wedderburn's theorem.

For an irreducible division algebra (s) of matrices, the number $h=k / n$ must be equal to 1 ; as follows for instance from (9.1C) since here it is certainly impossible to choose the coefficients in two rows arbitrarily. For such a $\$ 5$ the rank $k$ and the degree $n$ are equal.

If we apply this to $\mathfrak{T}$ and $\mathfrak{W}$ in $(9.3 \mathrm{~B})$, we have $z=t$ and $v=t$ and hence

$$
k v=n^{2},
$$

where $n$ is the degree of the irreducible semigroup $\$$, $k$ is the rank of $\$$, and $v$ the rank of $\mathfrak{S}(\mathfrak{S})$. This is the generalized Burnside theorem. We obtain the original theorem when we assume that the field $K$ is algebraically closed, and therefore $v=1$, i.e., $k=n^{2}$. This can also be derived from (9.2A),

We also obtain

(9.4A) If $K$ is a field, and (s) an irreducible algebra of matrices, we have $\mathfrak{S}(\mathbb{S}(\$))=(\$)$.

Proof. We have $\mathfrak{M}(\mathfrak{S})=\mathbb{S}$, and, because of the commutativity of $K$, this is not affected by a similarity transformation. We may assume $B$ in the form $\mathfrak{S}=[\mathfrak{T}]_{s}$. Further, $\mathfrak{M}(\mathfrak{T})=\mathfrak{T}$. Then $(9.3 \mathrm{~A})$ in connection with $(6.2 \mathrm{~B})$ gives $\mathfrak{S}(\mathfrak{S}(\mathfrak{S}))=\mathfrak{S}(s \times \mathfrak{W})=[\mathfrak{S}(\mathfrak{W})]_{s}=[\mathfrak{T}]_{s}=(\mathfrak{S}$. The same equation $\mathfrak{S} \mathfrak{S}(\mathfrak{S})=\mathbb{S}$ must have been true then, before $(\$)$ was subjected to the similarity transformation mentioned in $(9.3 \mathrm{~A})$.

10. ON THE REPRESENTATION OF SETS OF MATRICES AS DIRECT SUMS.

\section{THE RADICAL}

1. We say that a set $\mathfrak{Q}$ of square matrices of degree $n$ is the sum of two subsets $\mathfrak{A}$ and $\mathfrak{B}$, if $\mathfrak{Q}$ consists of all the matrices $A+B$ with $A$ in $\mathfrak{A}, B$ in $\mathfrak{B}$. We write $\mathfrak{Q}=\mathfrak{A} \oplus \mathfrak{B}$, if, besides, we have $\mathfrak{A} \mathfrak{B}=0$ and $\mathfrak{B} \mathfrak{A}=0$ (i.e., $A B=0$ and $B A=0$ for any $A$ in $\mathfrak{A}$ and any $B$ in $\mathfrak{B})\left({ }^{34}\right)$. We first prove

(10.1A) If the semigroup (s) breaks up completely into $m$ distinct (i.e., nonsimilar) irreducible constituents

$$
\mathbb{B}=\left(\begin{array}{llll}
\mathfrak{F}_{1} & & & \\
& \mathfrak{F}_{2} & & \\
& & \cdot & \\
& & \cdot & \mathfrak{F}_{m}
\end{array}\right),
$$

${ }^{(34)}$ The notation here is different from that in $\$ 4.1$. 
then the l-rank of \&5 is equal to the sum $k_{1}+k_{2}+\cdots+k_{m}$ of the l-ranks $k_{i}$ of $\mathfrak{F}_{i}$.

This is a generalization of the Frobenius-Schur theorem $\left({ }^{35}\right)$.

Proof. We can find an $l$-basis of $\mathfrak{M}(\mathfrak{S})$ such that for a fixed $\nu$ the last $k_{\nu}$ basis elements have $k_{\nu} l$-independent matrices in the place of $\mathfrak{F}_{\nu}$. After subtracting a suitable linear combination of these basis elements from the first $k-k_{\nu}$ basis elements, we may assume that the latter have 0 in the place of $\mathfrak{F}_{\nu}$. On forming the regular representation $\Re$ of $B$ with regard to this basis, we obtain

$$
\Re=\left(\begin{array}{ll}
* & \\
* & \Re_{\nu}
\end{array}\right)
$$

where $\Re_{\nu}$ is the regular representation of $\mathfrak{F}_{\nu}$. If $f_{\nu}$ is the degree of $\mathfrak{F}_{\nu}$, we have $\Re_{\nu} \cong\left(k_{\nu} / f_{\nu}\right) \times \mathfrak{F}_{\nu}$ (cf. (9.1A)). The constituents $\mathfrak{F}_{\nu}$ of $\Re$ occupy therefore at least $k_{\nu}$ ordinary rows and columns of $\Re$. The degree $k$ of $\Re$, then, cannot be smaller than the sum of all the $k_{\nu}$. On the other hand, (37) shows that $k \leqq k_{1}+\cdots+k_{m}$, and this proves the statement. At the same time, we see

(10.1B) The regular representation $\Re$ of $\$$ in (10.1A) contains the constituent $\mathfrak{F}_{\nu}$ with the multiplicity $k_{\nu} / f_{\nu}$, where $f_{\nu}$ is the degree of $\mathfrak{F}_{\nu}$.

The result (10.1A) can be formulated in the following manner:

(10.1C) Under the assumption of (10.1A), the module $\mathfrak{M}(\mathfrak{B})$ is a direct sum $\mathfrak{M}(\mathfrak{S})=\mathfrak{U}_{1} \oplus \mathfrak{U}_{2} \oplus \cdots \oplus \mathfrak{U}_{m}$ where $\mathfrak{H}_{\mu}$ consists of those matrices $\mathfrak{M}(\mathfrak{S})$ which have nonzero elements only in the place of the constituent $\mathfrak{M}\left(\mathfrak{F}_{\mu}\right)$ of $\mathfrak{M}(\mathfrak{S})$.

Proof. Let $M_{\mu}$ be an arbitrary element of $\mathfrak{M}\left(\mathfrak{F}_{\mu}\right)(\mu=1,2, \cdots, m)$, and set

$$
\bar{M}=\left(\begin{array}{llll}
M_{1} & & & \\
& M_{2} & & \\
& & \ddots & \\
& & & M_{m}
\end{array}\right) .
$$

All these $\bar{M}$ form a $(K, \mathfrak{S})$-double module $\overline{\mathfrak{M}}$. We have $\overline{\mathfrak{M}} \supseteq \mathfrak{M}(\mathfrak{S})$, and both these modules have the same $l$-rank according to $(10.1 \mathrm{~A})$. Hence $\overline{\mathfrak{M}}=\mathfrak{M}(\mathfrak{S})$. We now choose $M_{\mu}$ arbitrarily in $\mathfrak{M}\left(\mathfrak{F}_{\mu}\right)$, and $M_{\nu}=0$ for $\nu \neq \mu$. The corresponding $\bar{M}$ form a submodule $\mathfrak{U}_{\mu}$ of $\mathfrak{M}(\mathfrak{S})$, and $\mathfrak{M}(\$)$ is the direct sum $\mathfrak{U}_{1} \oplus \cdots \oplus \mathfrak{U}_{m}$.

2. In order to study further the decomposition into direct sums, we consider two sets of square matrices $\mathfrak{A}$ and $\mathfrak{B}$ of the same degree $n$, such that $\mathfrak{A} \mathfrak{B}=0$. Let $\mathfrak{B}$ be the space in which the transformations of $\mathfrak{A}$ and $\mathfrak{B}$ take place. Let $\mathfrak{B}_{0}$ be the subspace consisting of those vectors $V_{0}$ for which $\mathfrak{A} V_{0}=0$. Then we have $\mathfrak{B} \mathfrak{B} \subseteq \mathfrak{B}_{0}$. If $\mathfrak{B}_{0}$ has $s$ dimensions and we choose a basis of $\mathfrak{B}$

(35) Frobenius-Schur [10]. 
in which the last $s$ vectors form a basis of $\mathfrak{B}_{0}$, then in the corresponding similar set $P^{-1} \mathfrak{A} P$ the last $s$ columns consist of zeros, and in $P^{-1} \mathfrak{B} P$ the first $s$ rows consist of zeros. Hence

(10.2A) If $\mathfrak{A}$ and $\mathfrak{B}$ are two sets of square matrices of degree $n$ and $\mathfrak{A} \mathfrak{B}=0$, then we can find a similarity transformation $P$ such that

$$
P^{-1 \mathfrak{A} P}=\left(\begin{array}{ll}
\mathfrak{A}_{1} & \\
\mathfrak{C} & 0
\end{array}\right), \quad P^{-1 \mathfrak{B} P}=\left(\begin{array}{ll}
0 & \\
\mathfrak{D} & \mathfrak{B}_{1}
\end{array}\right)
$$

where both sets are broken up according to the same scheme $(n-s, s \mid n-s, s)$.

We may have here $s=0$, if $\mathfrak{B}=0$. Then the second row and column in (38) are missing. Similarly, we may have $s=n$, if $\mathfrak{A}=0$, and then the first row and column in (38) are missing. If $\mathfrak{A} \neq 0, \mathfrak{B} \neq 0$, the set $\mathfrak{Q}$ consisting of all sums $A+B$ with $A$ in $\mathfrak{A}, B$ in $\mathfrak{B}$ is reducible. This gives

(10.2B) The set $\mathfrak{U}_{\mu}$ in $(10.1 \mathrm{C})$ cannot be written as a direct sum $\mathfrak{A} \oplus \mathfrak{B}$ with $\mathfrak{A} \neq 0, \mathfrak{B} \neq 0$.

3. As an application of $(10.2 \mathrm{~A})$, we prove

(10.3A) Let $\mathfrak{Q}$ be a set of square matrices of degree $n$ which has no constituents 0 . If $\mathfrak{Q}$ can be written as a sum $\mathfrak{Q}=\mathfrak{A} \oplus \mathfrak{B}$ with $\mathfrak{A} \neq 0, \mathfrak{B} \neq 0$, then there exists a similarity transformation $P$ such that

$$
P^{-1} \mathfrak{Q P}=\left(\begin{array}{ll}
\mathfrak{A}_{1} & \\
& \mathfrak{B}_{1}
\end{array}\right)
$$

and $P^{-1 \mathfrak{A} P}$ consists of the matrices of $P^{-1} \mathfrak{Q} P$ which have 0 in the place of $\mathfrak{B}_{1}$ and $P^{-1} \mathfrak{B P}$ consists of those matrices of $P^{-1} \mathfrak{Q P}$ which have 0 in the place of $\mathfrak{A}_{1}$.

Proof. We may determine $P$ such that $P^{-1 \mathfrak{A} P}$ and $P^{-1} \mathfrak{B} P$ have the form (38). The set $\mathfrak{B}_{1}$ has no constituent 0 , since otherwise 0 would also be a constituent of the sum of the two sets (38), and hence of $\mathfrak{Q}$. If $\mathfrak{S}$ is the semigroup generated by $P^{-1} \mathfrak{B P}$, and $\mathfrak{M}(\mathfrak{S})$ its enveloping module, then the matrices $M$ of $\mathfrak{M}(\mathfrak{S})$ are $l$-annihilators of $P^{-1} \mathfrak{Q} P$. Further $\mathfrak{M}(\mathfrak{S})$ breaks up in the same form as $P^{-1} \mathfrak{B P}$ in (38) the first constituent being 0 and the second $\mathfrak{M}\left(\mathfrak{S}_{1}\right)$ where $\mathfrak{S}_{1}$ is the semigroup generated by $\mathfrak{B}_{1}$. According to $(8.2 \mathrm{G})$ this set $\mathfrak{M}\left(\mathfrak{S}_{1}\right)$ contains the unit matrix $I$. Let $J$ be a matrix of $\mathfrak{M}(\mathfrak{S})$ which has $I$ in the place of $\mathfrak{M}\left(\mathfrak{S}_{1}\right)$, and let $A$ be an arbitrary element of $\mathfrak{A}$. We set

$$
J=\left(\begin{array}{ll}
0 & \\
D & I
\end{array}\right), \quad P^{-1} A P=\left(\begin{array}{ll}
A_{1} & \\
C & 0
\end{array}\right) .
$$

Because of $J\left(P^{-1} A P\right)=0$, we have $D A_{1}+C=0$. We subtract the first row in (38), $l$-multiplied by $D$, from the second row and add the second column, 
$r$-multiplied by $D$, to the first column. This amounts to a similarity transformation (cf. (3.3A)). Afterwards we have $\mathfrak{E}=0$, and we may assume that this is also true in (38). Then $\mathfrak{B P}=0$ implies $\mathfrak{D A}_{1}=0$. Since $\mathfrak{A}_{1}$ also has no constituent 0 , it follows that $\mathfrak{D}=0$, and this proves the statement.

Repeated application of (10.3A) gives

(10.3B) If a set $\mathfrak{Q}$ without constituent 0 is a direct sum $\mathfrak{U}_{1} \oplus \cdots \oplus \mathfrak{U}_{m}$ $\left(\mathfrak{U}_{\mu} \neq 0\right)$, then, after a suitable similarity transformation $P, P^{-1} \mathfrak{Q} P$ splits completely into $m$ constituents and the matrices of $P^{-1} \mathfrak{u}_{\mu} P$ have coefficients not equal to 0 only at the place of the $\mu$ th of these constituents.

4. The radical $\mathfrak{N}$ of a set $\mathfrak{A}$ of square matrices consists of those matrices $N$ of $\mathfrak{A}$ which are represented by 0 in every irreducible constituent of $\mathfrak{A}$. Then $Q$ is also represented by 0 in the Loewy constituents $L_{i}(\mathfrak{A})$; i.e., $N$ has zeros in the main diagonal in (14). A simple computation shows that the product of any $L(\mathfrak{U})$ matrices vanishes. If $\mathfrak{A}$ is a ring of matrices, $\mathfrak{N}$ is a nilpotent ideal, $\mathfrak{N}^{L}=0$, for $L=L(\mathfrak{U})$.

We can easily study the radical of the enveloping module $\mathfrak{M}(\mathfrak{S})$ of a semigroup $\$$, provided that $\$$ has been brought into a suitable form by a similarity transformation.

(10.4A) Let (I) be a semi-group which splits into irreducible constituents

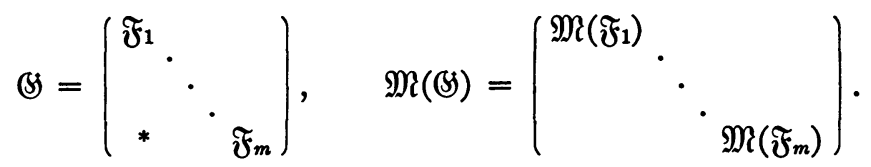

Then the radical $\mathfrak{R}$ of $\mathfrak{M}(\mathfrak{S})$ has at least the l-rank $k-\lambda$ where $k$ is the l-rank of $(\$)$ and $\lambda$ the degree of the first Loewy constituent $\mathfrak{R}_{1}(\Re)$ of the regular representation $\Re$ of $\$$.

Proof. Let $M_{1}, \cdots, M_{k}$ be an $l$-basis of $\mathfrak{M}(())$ with regard to which the regular representation $\Re$ appears in its lower Loewy normal form. If $G$ is an arbitrary element of $(\$)$, we have

$$
M_{\kappa} G=\sum r_{\kappa \lambda} M_{\lambda}
$$

where $R=\left(r_{k \lambda}\right)$ is the matrix of $\Re$, associated with $G$. If $\mathfrak{B}$ is one of the $\mathfrak{F}_{k}$, and $M_{\kappa}$ corresponds to $U_{\kappa}$ in $\mathfrak{M}(\mathscr{B})$ and $G$ corresponds to $B$, we have

$$
U_{k} B=\sum r_{k \lambda} U_{\lambda}
$$

We now apply (7.8A) setting $U_{\kappa}=\left(h_{\alpha \beta}^{(\alpha)}\right)$. Then $P_{\mu}=\left(h_{\mu \beta}^{(\alpha)}\right)$ will intertwine $\Re$ and $\mathscr{B}$. Because of $(5.2 \mathrm{~A})$, only the last $\lambda$ rows of $P_{\mu}$ contain coefficients not equal to 0 . Hence

$$
h_{\mu \beta}^{(\alpha)}=0 \quad \text { for } \alpha \leqq k-\lambda .
$$


This shows $U_{k}=0$ for $\kappa \leqq k-\lambda$. Hence $M_{1}, \cdots, M_{k-\lambda}$ are represented by 0 in each $\mathfrak{M}\left(\mathfrak{F}_{\mu}\right)$ and, therefore, belong to $\mathfrak{N}$.

(10.4B) If the semigroup (5) splits into irreducible constituents and the radical of $\mathfrak{M}(\mathbb{S})$ vanishes, then $(\mathbb{S}$ is completely reducible.

Proof. We have here $k=\lambda$; i.e., $\Re$ is completely reducible, $L(\Re)=1$. We denote by $\overline{(S)}$ the set obtained from $(S)$ by replacing everything below the main diagonal in (39) by 0 's, and omitting all constituents 0 . According to (8.2G), a suitable linear combination of the elements of $\bar{\Theta}$ is equal to the unit matrix. A corresponding linear combination of the elements of $(S)$ gives a matrix $J$ of $\mathfrak{M}(\mathfrak{S})$ which in (39) has a unit matrix in the place of every $\mathfrak{M}\left(\mathfrak{F}_{\rho}\right) \neq 0$ and, of course, 0 in the place of every $\mathfrak{M}\left(\mathfrak{F}_{\rho}\right)=0$. The product $J G$ of $J$ with an element $G$ of $B$ has the same main diagonal as $G$. Then $G-J G$ lies in the radical of $\mathfrak{M}(\mathfrak{S})$, and hence $J G=G$. Now $(8.2 \mathrm{~F})$ can be applied. We obtain $L(\$)=L(\Re)=1$, i.e., (S) is completely reducible.

If $K$ is noncommutative, the converse of assertion (10.4B) need not be true.

5. Repeated application of (10.2A) now gives

(10.5A) If a set $\mathfrak{Q}$ of square matrices is a sum of sets $\mathfrak{Q}_{1}, \mathfrak{Q}_{2}, \cdots, \mathfrak{Q}_{r}$, if $\mathfrak{Q}_{i} \mathfrak{Q}_{j}=0$ for $i<j$, and if no $\mathfrak{Q}_{i}$ lies in the radical of $\mathfrak{Q}$, then, after a similarity transformation, $\mathfrak{Q}$ will split into $r$ constituents $\mathfrak{T}_{1}, \mathfrak{T}_{2}, \cdots, \mathfrak{T}_{r}$. The matrices of $\mathfrak{Q}_{\rho}$ have 0 in the place of every $\mathfrak{I}_{\sigma}, \sigma \neq \rho$.

Proof. We apply $(10.2 \mathrm{~A})$ to the case that $\mathfrak{A}$ is the sum of $\mathfrak{Q}_{1}, \cdots, \mathfrak{Q}_{r-1}$ and $\mathfrak{B}=\mathfrak{Q}_{r}$. We then have an equation (38). Here, $\mathfrak{B}_{1} \neq 0$, since $\mathfrak{Q}_{r}$ does not lie in the radical of $\mathfrak{Q}$. Let $\mathfrak{Q}_{i}{ }^{*}$ be the set which stands in $\mathfrak{Q}_{i} \subseteq \mathfrak{Q}$ in the place of $\mathfrak{A}_{1}(i=1,2, \cdots, r-1)$. Then $\mathfrak{A}_{1}$ is the sum of $\mathfrak{Q}_{1}^{*}, \cdots, \mathfrak{Q}_{r-1}^{*}$, and $\mathfrak{Q}_{i}^{*}$ does not belong to the radical of $\mathfrak{A}_{1}$, since otherwise $\mathfrak{Q}_{i}$ would belong to the radical of $\mathfrak{Q}$. If the theorem is true for the sums of $r-1$ sets, it now follows for the sum of $r$ sets.

\section{Rings Which CONTAIN $n \times K$}

1. We now consider rings of matrices $\mathfrak{A}$ of degree $n$ with coefficients in the division ring $K$ which are at the same time $K$-left modules and $K$-right modules, i.e., which contain $\gamma A$ and $A \gamma$ for all $A$ in $\mathfrak{A}$ and all $\gamma$ in $K$. Of course, this property will not always be preserved under similarity transformations of $\mathfrak{A}$.

If $\mathfrak{A}$ is a ring which is a $K$-left module, we have $\mathfrak{M}(\mathfrak{A})=\mathfrak{A}$ in the notation of $\$ 8.1$. If $\mathfrak{A}$ has no constituent 0 , then $\mathfrak{A}$ contains the unit matrix according to $(8.2 \mathrm{G})$, and hence all the matrices $\gamma I, \gamma$ in $K$. These matrices form a set $\Omega$ isomorphic with $K$ which we may denote by $n \times K$, if we identify the matrix $(\gamma)$ of first degree with $\gamma$. Any ring $\mathfrak{A}$ which contains $\Re=n \times K$ is a $K$-left module and a $K$-right module. 
We prove several lemmas which connect $\mathfrak{A}$ with sets of matrices whose coefficients lie in the centre $Z$ of $K$. This centre $Z$ is a field.

(11.1A) If $\mathfrak{A}$ is a ring of matrices which is a $K$-left module and a $K$-right module, then an l-basis $A_{1}, A_{2}, \cdots, A_{k}$ can be chosen such that the coefficients of each $A_{\kappa}$ lie in the centre $Z$ of $K$. The $A_{\kappa}$ form a basis of the algebra $\overline{\mathfrak{A}}=\mathfrak{A} \cap[Z]_{n}$ over the field $Z$. We have $\mathfrak{A}=\mathfrak{M}(\overline{\mathfrak{A}})$.

Proof. The set $\mathfrak{A}$ obviously is a $(K, \Re)$-double module. Now $\Re=n \times K$ is completely reducible with $K$ as its only irreducible constituent. According to (7.6A), the same is true for the set $\Omega^{*}$ which $\mathfrak{A}$ associates with $\Omega$. If we choose a suitable $l$-basis $A_{\kappa}$ in $\mathfrak{A}$, we have $\mathfrak{\Omega}^{*}=k \times K$ where $k$ is the $l$-rank of $\mathfrak{A}$. Then $A_{\kappa} \gamma=\gamma A_{\kappa}$ for every $\gamma$ in $K$. This shows that the coefficients of $A_{\kappa}$ lie in $Z$.

Every element $A$ of $\mathfrak{A}$ has the form $A=\sum \gamma_{\kappa} A_{\kappa}$ with coefficients $\gamma_{\kappa}$ in $K$. These $\gamma_{\kappa}$ are uniquely determined, and we have a system of $n^{2}$ linear equations for them. If $A$ belongs to $[Z]_{n}$, i.e., if the coefficients of $A$ lie in $Z$, then the coefficients of these linear equations lie in $Z$. Hence (cf. §3.4) the $\gamma_{\kappa}$ themselves lie in $Z$. This proves (11.1A).

We now consider the commuting ring $\mathfrak{S}(\mathfrak{A})$. We prove

(11.1B) If $\mathfrak{A}$ is a ring of matrices which is a $K$-left module and has no constituent 0 , then $\mathfrak{S}(\mathfrak{Q})=\mathfrak{S}(\overline{\mathfrak{A}}) \cap[Z]_{n}$ and $\mathfrak{S}(\overline{\mathfrak{A}})=\mathfrak{M}(\mathfrak{S}(\mathfrak{A}))$.

Proof. Here, $\mathfrak{R}=n \times K \subseteq \mathfrak{A}$ and hence $\mathfrak{S}(\mathfrak{U}) \subseteq \mathfrak{S}(n \times K)=[\mathfrak{S}(K)]_{n}=[Z]_{n}$. Further $\mathfrak{S}(\mathfrak{A}) \subseteq \mathfrak{C}(\overline{\mathfrak{A}})$. On the other hand, every matrix $M$ of the intersection $\mathfrak{S}(\overline{\mathfrak{A}}) \cap[\mathfrak{B}]_{n}$ commutes with the $A_{\kappa}$ of $(11.1 \mathrm{~A})$ and with all $\gamma$ in $K$. Hence $M$ belongs to $\mathfrak{S}(\mathfrak{U})$, and $\mathfrak{S}(\mathfrak{A})=\mathfrak{C}(\overline{\mathfrak{Q}}) \cap[Z]_{n}$. The ring $\mathfrak{S}(\overline{\mathfrak{U}})$ contains $\mathfrak{\Re}$. If we apply $(11.1 \mathrm{~A})$ to it, we obtain $\mathfrak{S}(\overline{\mathfrak{A}})=\mathfrak{M}(\mathfrak{S}(\mathfrak{U}))$.

2. (11.2A) If $\mathfrak{A}$ is a set of matrices of degree $n$ which contains $\Re=n \times K$, we may determine a matrix $P$ with coefficients in the centre $Z$ of $K$, such that $P^{-1 \mathfrak{A} P}=\mathfrak{A}^{*}$ splits into irreducible constituents. If $\mathfrak{A}$ is completely reducible, we may add here the additional condition that $\mathfrak{A}$ splits completely into irreducible constituents.

Proof. We split $\mathfrak{A}$ into irreducible constituents using a similarity transformation $Q$ with coefficients in $K$,

$$
Q^{-1 \mathfrak{A} Q}=\left(\begin{array}{lll}
\mathfrak{A}_{1} & & \\
& \cdot & \\
& \cdot & \\
& & \mathfrak{A}_{m}
\end{array}\right) .
$$

If $\mathfrak{A}$ is completely reducible, we may assume that all the terms below the main diagonal vanish. The subset $Q^{-1} \Re Q$ of $Q^{-1 \mathfrak{A} Q}$ is completely reducible, and $K$ is its only irreducible constituent. If we use (40) only for $Q^{-1} \Re Q$, the set $\Omega_{\mu}$ 
which takes the place of $\mathfrak{A}_{\mu}$ is completely reducible, and $K$ is its only irreducible constituent. After applying a suitable similarity transformation to (40), we may assume that $\Re_{\mu}=f_{\mu} \times K$ where $f_{\mu}$ is the degree of $\mathfrak{A}_{\mu}$. Now the set $Q^{-1} \Re Q$ splits into $n$ constituents $K$. Since it is completely reducible, we can transform it into $n \times K$ by elementary similarity transformations $\left({ }^{36}\right), \S 4.5$. If we apply these elementary similarity transformations to (40), the triangular form will not be changed. We may therefore assume right from the beginning that $Q^{-1} \Re Q=n \times K=\Re$. If $\mathfrak{A}$ is completely reducible, no elementary similarity transformations are needed. We now have $Q^{-1}(\gamma I) Q=\gamma I$ for every $\gamma$ in $K$. Then $\gamma Q=Q \gamma$, i.e., $Q$ has coefficients in $Z$, and we may take $P=Q$.

3 . Let $\mathfrak{A}$ be a ring of matrices of degree $n$ which contains $n \times K$. If $\mathfrak{B}$ is a homomorphic set of matrices of degree $m$, and if the element $\gamma I_{n}$ of $\mathfrak{A}$ corresponds to $\gamma I_{m}$ in $\mathscr{B}$ for every $\gamma$ in $K$, then $\mathfrak{B}$ is said to be a representation of degree $m$ of $\mathfrak{A}$. If we split $\mathfrak{A}$ in to irreducible constituents by means of the transformation $P$ of $(11.2 \mathrm{~A})$, the irreducible constituents of $\mathfrak{A}$ will then be representations of $\mathfrak{A}$.

If we use the basis $A_{\kappa}$ of (11.1A) for the definition of the regular representation $\Re$ of $\mathfrak{A}$, then $\Re$ will actually be a representation of $\mathfrak{A}$.

Any two representations $\mathfrak{B}_{1}$ and $\mathfrak{B}_{2}$ of $\mathfrak{A}$ are to be considered as related sets (§3.1) with $\mathbb{Z}=\mathfrak{A}$. If $\mathfrak{B}_{1}$ and $\mathfrak{B}_{2}$ are similar, say $\mathfrak{B}_{1}=Q^{-1} \mathfrak{B}_{2} Q$, then $\gamma I=Q^{-1}(\gamma I) Q$ for every $\gamma$ in $K$. This implies that $Q$ has coefficients in $Z$.

(11.3A) If two representations of the ring $\mathfrak{A} \supseteq n \times K$ are similar, then the corresponding similarity transformation has coeffcients in the centre of $K$.

4. We now derive the results of the structure theory of algebras $\left({ }^{37}\right)$.

(11.4A) If $\mathfrak{A} \neq 0$ is an irreducible ring of matrices which is a $\mathrm{K}$-left module, then $\mathfrak{A} \sim[\mathfrak{T}]_{s}$ where $\mathfrak{T}$ is a division ring consisting of matrices and $s>0$ an integer. We have $\mathfrak{S}(\mathfrak{S}(\mathfrak{U}))=\mathfrak{A}$.

Proof. Since $\mathfrak{A}$ has no constituent 0 , we have $\Re=n \times K \subseteq \mathfrak{A}$. Obviously, $\mathfrak{C}(\mathfrak{S}(\mathfrak{A})) \supseteq \mathfrak{A} \supseteq \Re$. On applying $(11.1 \mathrm{~A})$ to this ring $\mathfrak{S}(\mathfrak{S}(\mathfrak{A}))$ we see that it has a basis consisting of matrices $C_{\rho}$ with coefficients in $Z$. These matrices $C_{\rho}$ have the following two properties: (a) they belong to $[Z]_{n}$; (b) they commute with every element of $\mathfrak{S}(\overline{\mathfrak{A}}) \cap[Z]_{n}$, which is equal to $\mathfrak{S}(\mathfrak{A})$ because of $(11.1 \mathrm{~B})$.

From (11.1A) it follows that $\overline{\mathfrak{A}}$ is irreducible with regard to $Z$. Let us consider for the moment only matrices with coefficients in $Z$. Then (9.4A) shows that the commuting ring of the commuting ring of $\overline{\mathfrak{A}}$ is $\overline{\mathfrak{A}}$ itself. In other words: every matrix $C$ with the properties (a) and (b) belongs to $\overline{\mathfrak{A}}$. Then the $C_{\rho}$ belong to $\overline{\mathfrak{A}} \subseteq \mathfrak{A}$ and hence $\mathfrak{S}(\mathfrak{S}(\mathfrak{A})) \subseteq \mathfrak{A}$ which implies $\mathfrak{S}(\mathfrak{S}(\mathfrak{A}))=\mathfrak{A}$.

We can now use the argument of $\S 9.3$. We set $\mathfrak{B}=\mathfrak{E}(\mathfrak{A})$; this set is com-

(36) The degrees $n_{\lambda}$ in $\$ 3.3$ are here to be taken as equal to 1 .

$\left.{ }^{37}\right)$ Cf., for instance, Albert [1, 2], Deuring [7]. 
pletely reducible and has only one irreducible constituent $\mathfrak{B}$. We may set

$$
Q^{-1 \mathfrak{B} Q}=s \times \mathfrak{B}
$$

where $Q$ is a matrix with coefficients in $K$ (not necessarily in $Z$ ), and $\mathfrak{W}$ is irreducible and a division ring. Then

$$
Q^{-1 \mathfrak{Q} Q}=\left(\mathfrak{S}(s \times \mathfrak{B})=[\mathfrak{G}(\mathfrak{W})]_{s},\right.
$$

and $\mathfrak{S}(\mathfrak{B})=\mathfrak{T}$ itself is irreducible, and a division ring. This proves (11.4A).

We now prove easily in the familiar manner that the ring $\mathfrak{A}$ is simple, i.e., possesses no proper subideal. There is no properly nilpotent element not equal to 0 in $\mathfrak{A}$.

Consider an arbitrary ring $\mathfrak{A}$ of matrices which contain $n \times K$. We determine a similarity transformation with the properties stated in (11.2A). Since the elements of $n \times K$ are transformed into themselves, we may assume without restriction that $\mathfrak{A}$ itself splits into irreducible constituents,

$$
\mathfrak{A}=\left(\begin{array}{lll}
\mathfrak{A}_{1} & & \\
& \ddots & \\
& & \mathfrak{A}_{r}
\end{array}\right) .
$$

Using (11.3A), we easily see that we may assume that similar $\mathfrak{A}_{\rho}$ are always equal. Let $\mathfrak{F}_{1}, \mathfrak{F}_{2}, \cdots, \mathfrak{F}_{m}$ be the distinct irreducible constituents appearing, and denote the $l$-rank of $\mathfrak{F}_{\mu}$ by $k_{\mu}$. Then $\mathfrak{M}(\mathfrak{A})=\mathfrak{A}, \mathfrak{M}\left(\mathfrak{F}_{\mu}\right)=\mathfrak{F}_{\mu}$.

If we replace everything below the main diagonal in (41) by 0 , we obtain a representation $\mathfrak{A}^{*}$ of $\mathfrak{A}$. The elements of the radical $\mathfrak{N}$ of $\mathfrak{A}$ and only these are represented by 0 ; we see that $\mathfrak{A} / \mathfrak{N}$ and $\mathfrak{A}^{*}$ are isomorphic. From (10.1A) it follows that $\mathfrak{I}^{*}$ has the $l$-rank $\sum k_{\mu}$. Hence

(11.4B) If $\mathfrak{A}$ is a ring of matrices which contains $n \times K$, its l-rank is given by $k=k_{1}+k_{2}+\cdots+k_{m}+\nu$ where $k_{1}, k_{2}, \cdots, k_{m}$ are the l-ranks of the nonsimilar irreducible constituents $\mathfrak{F}_{1}, \mathfrak{F}_{2}, \cdots, \mathfrak{F}_{m}$ of $\mathfrak{A}$ and $\nu$ is the l-rank of the radical $\mathfrak{N}$ of $\mathfrak{A}$. If in each $\mathfrak{F}_{\mu}$ an arbitrary element $F_{\mu}$ has been chosen, then there are elements $A$ of $\mathfrak{A}$ which are represented by $F_{\mu}$ in $\mathfrak{F}_{\mu}$ for $\mu=1,2, \cdots, m$.

If $\mathfrak{A}$ is completely reducible, we may assume that (41) splits completely into irreducible constituents. We then find $\mathfrak{N}=0$. Conversely, if $\mathfrak{N}=0$, it follows from (10.4B) that $\mathfrak{A}$ is completely reducible. A ring is semisimple, if its radical vanishes. Hence

(11.4C) A ring $\mathfrak{A} \supseteq n \times K$ is semisimple, if and only if $\mathfrak{A}$ is completely reducible.

Ordinarily, the radical is defined as the set of all properly nilpotent elements $N$ of $\mathfrak{A}$. But to such an $N$, there corresponds a properly nilpotent $N_{\rho}$ of $\mathfrak{F}_{\rho}$. Since $\mathfrak{F}_{\rho}$ is irreducible, we have $N_{\rho}=0$. Hence $N$ belongs to $\mathfrak{N}$. Con- 
versely, every element of $\mathfrak{N}$ is properly nilpotent. Both definitions of the radical coincide.

The rings $\mathfrak{A} / \mathfrak{N}$ and $\mathfrak{A} *$ were isomorphic. Hence

(11.4D) If the ring $\mathfrak{A} \supseteq n \times K$ has the radical $\mathfrak{R}$, then $\mathfrak{A} / \mathfrak{N}$ is semisimple.

If $\mathfrak{A}$ is semisimple, we can apply (10.1C) and find:

(11.4E) Let $\mathfrak{A} \supseteq n \times K$ be a semisimple ring. If $\mathfrak{F}_{1}, \mathfrak{F}_{2}, \cdots, \mathfrak{F}_{m}$ are the nonsimilar irreducible constituents of $\mathfrak{A}$, then $\mathfrak{A}$ is the direct sum $\mathfrak{A}=\mathfrak{U}_{1} \oplus \mathfrak{U}_{2} \oplus \ldots$ $\oplus \mathfrak{U}_{m}$ of $m$ simple rings and $\mathfrak{U}_{\mu}$ is isomorphic with $\mathfrak{F}_{\mu}$.

On combining the last part of $(11.4 \mathrm{~A})$ with $\S 6.2$, we obtain

(11.4F) If $\mathfrak{A}$ is a completely reducible ring which contains $n \times K$, then $\mathfrak{S}(\mathfrak{S}(\mathfrak{A}))=\mathfrak{A}$.

Finally, we can show that

(11.4G) If $\mathfrak{B}$ is a simple ring of matrices, and $\mathfrak{B} \supseteq n \times K$, then $\mathfrak{B} \sim t \times \mathfrak{A}$, where $t>0$ is an integer and $\mathfrak{A}$ an irreducible ring. Then $\mathfrak{B}$ is isomorphic to the ring $\mathfrak{A}$ whose structure is described by (11.4A).

Proof. The radical of $\mathfrak{B}$ must vanish. Therefore, $\mathfrak{B}$ is completely reducible. From (11.4E) it follows that $\mathfrak{B}$ has only one irreducible constituent.

5. We consider an arbitrary ring $\mathfrak{A}$ of matrices which contains $n \times K$ and a representation $\mathfrak{B}$ of $\mathfrak{A}$. Let $A_{1}, \cdots, A_{k}$ be an $l$-basis of $\mathfrak{A}$ and $A$ an arbitrary element of $\mathfrak{A}$. The regular representation $\Re$ is defined by

$$
A_{\kappa} A=\sum_{\lambda} r_{k \lambda} A_{\lambda}, \quad \quad r_{\kappa \lambda} \text { in } K .
$$

If $A_{\kappa} \rightarrow B_{\kappa}, A \rightarrow B$ are the associated elements in $\mathfrak{B}$, we find

$$
B_{\kappa} B_{\kappa}=\sum r_{\kappa \lambda} B_{\lambda} \text {. }
$$

We may assume that for a certain $t$ the elements $B_{1}=\cdots=B_{t}=0$ and that $B_{t+1}, \cdots, B_{k}$ are $l$-independent. On comparing (42) and (43), we see that the regular representation of $\mathfrak{B}$ appears as an end constituent of $\Re$. Using (8.2A) we now find:

(11.5A) Let $\mathfrak{A}$ be a ring of matrices containing $n \times K$. Every representation $\mathfrak{B}$ of $\mathfrak{A}$ of degree $m$ appears as an end constituent of $m \times \Re$ where $\mathfrak{R}$ is the regular representation of $\mathfrak{A}$. Further, $\mathfrak{B}$ appears as a constituent of $m n \times \mathfrak{A}$.

As corollaries, we obtain:

(11.5B) If $\mathfrak{B}$ is a representation of $\mathfrak{A}$, then $L(\mathfrak{B}) \subseteq L(\mathfrak{A})$.

(11.5C) Every irreducible representation of $\mathfrak{A}$ appears as a constituent of $\mathfrak{A}$.

The following theorems are sometimes useful. 
(11.5D) If $\mathfrak{B}_{1}$ and $\mathfrak{B}_{2}$ are two representations of $\mathfrak{A}$ which have no irreducible constituent in common, then we can find an element $Q$ of $\mathfrak{A}$ which is represented by the unit matrix in $\mathfrak{B}_{1}$ and by 0 in $\mathfrak{B}_{2}$.

Proof. According to (11.4B), we can find an element $A$ of $\mathfrak{A}$ which is represented by the unit matrix in every irreducible constituent of $\mathfrak{B}_{1}$ and by 0 in every irreducible constituent of $\mathfrak{B}_{2}$. Then $A$ corresponds to a radical element $B_{2}$ of $\mathfrak{B}_{2}$. If we replace $A$ by a power of $A$, we may assume $B_{2}=0$. If $A$ is represented by $B_{1}$ in $\mathfrak{B}_{1}$, then $B_{1}-I$ lies in the radical of $\mathfrak{B}_{1}$; we have $\left(B_{1}-I\right)^{t}=0$ for some integer $t>0$. Hence we may write $I$ as a polynomial $f\left(B_{1}\right)$ without constant term of $B_{1}$. Then $Q=f(A)$ will satisfy the required conditions.

(11.5E) If $\mathfrak{B}_{1}$ and $\mathfrak{B}_{2}$ are two representations of $\mathfrak{A}$ which have no irreducible constituent in common, and if $B_{1}$ and $B_{2}$ are arbitrary elements of $\mathfrak{B}_{1}$ and $\mathfrak{B}_{2}$ respectively, then we may find an element $A$ of $\mathfrak{A}$ which is represented by $B_{1}$ in $\mathfrak{B}_{1}$ and by $B_{2}$ in $\mathfrak{B}_{2}$.

Proof. Let $A^{(1)}$ be an element of $\mathfrak{A}$ which is represented by $B_{1}$ in $\mathfrak{B}_{1}$ and determine $Q$ as in (11.5D). Then $Q A^{(1)}$ is represented by $B_{1}$ in $\mathfrak{B}_{1}$ and by 0 in $\mathfrak{B}_{2}$. Similarly, we may find an element $\bar{Q} A^{(2)}$ of $\mathfrak{A}$ which is represented by 0 in $\mathfrak{B}_{1}$ and by $B_{2}$ in $\mathfrak{B}_{2}$. Then we may set $A=Q A^{(1)}+\bar{Q} A^{(2)}$.

$(11.5 \mathrm{~F})$ If $\mathfrak{B}$ is a representation of $\mathfrak{A}$, the radical of $\mathfrak{A}$ is represented by the radical of $\mathfrak{B}$.

Proof. It is clear that radical elements of $\mathfrak{A}$ are represented by radical elements of $\mathfrak{B}$. Conversely, let $B$ be a radical element of $\mathfrak{B}$. We set $\mathfrak{B}_{1}=\mathfrak{B}$; for $\mathfrak{B}_{2}$ we take the representation of $\mathfrak{A}$ which splits completely into those irreducible representations of $\mathfrak{A}$ which do not appear in $\mathfrak{B}$. We then apply $(11.5 \mathrm{E})$ to the case $B_{1}=B, B_{2}=0$. The corresponding $A$ lies in the radical of $\mathfrak{A}$ and is represented by $B$ in $\mathfrak{B}$.

\section{THE REgUlaR REPRESENTATION OF RINGS WHICH CONTAIN $n \times K$}

1. We consider again a ring $\mathfrak{A}$ of matrices which is a $K$-left module, in particular a ring $\mathfrak{A}$ which contains $n \times K$. The regular representation $\mathfrak{R}$ of $\mathfrak{A}$ is a set of linear transformations of $\mathfrak{A}$ where $\mathfrak{A}$ is considered as a contragredient vector space. The element $A$ of $\mathfrak{A}$ is associated with the transformation $R(A)$ which maps the variable element $X$ of $\mathfrak{A}$ upon $X A=X^{*}$. In particular, if $A_{1}, A_{2}, \cdots, A_{k}$ is an $l$-basis of $\mathfrak{A}$, we have

where $R(A)=\left(r_{\kappa \lambda}\right)$.

$$
A_{\mathrm{k}}^{*}=A_{\mathrm{k}} A=\sum r_{\mathrm{k} \lambda} A_{\lambda}
$$

A subspace $\mathfrak{T}$ of $\mathfrak{A}$ which is invariant under the transformation of $\mathfrak{R}$, then, is a right ideal $\mathfrak{T}$ of $\mathfrak{A}$ which is a $K$-left module. Since we shall consider the elements $\gamma$ of $K$ as operators of $\mathfrak{A}$, where the operation is defined as $l$-multi- 
plication by $\gamma$, we shall tacitly assume that the right ideals considered are $K$-left modules.

Any splitting of $\Re$ into constituents will correspond to an ascending chain of invariant subspaces $\mathfrak{T}_{\nu}$ (cf. $\S 4.4$ ), i.e., an ascending chain of $r$-ideals of $\mathfrak{A}$. More explicitly, if

$$
\Re=\left(\begin{array}{lll}
\mathfrak{S}_{1} & & \\
& \ddots & \\
* & \ddots & \\
& & \mathfrak{S}_{m}
\end{array}\right)
$$

where $\mathfrak{S}_{\mu}$ is a constituent of degree $h_{\mu}$, then the linear combinations $\sum \gamma_{\nu} A_{\nu}$ of the first $h_{1}+h_{2}+\cdots+h_{\rho}$ basis elements with coefficients $\gamma_{\nu}$ in $K$ form an $r$-ideal $\mathfrak{T}_{\rho}$ of $\mathfrak{A}$. We set $\mathfrak{I}_{0}=(0)$ and have

$$
\mathfrak{T}_{0}=(0) \subset \mathfrak{T}_{1} \subset \mathfrak{I}_{2} \subset \cdots \subset \mathfrak{T}_{m-1} \subset \mathfrak{T}_{m}=\mathfrak{A} .
$$

Conversely, assume that such a chain of $r$-ideals is given where $\mathfrak{T}_{\mu}$ has the $l$-rank $t_{\mu}$. We choose the $l$-basis $A_{1}, A_{2}, \cdots, A_{k}$ of $\mathfrak{A}$ such that the first $t_{\mu}$ basis elements form an $l$-basis of $\mathfrak{T}_{\mu}$ for $\mu=1,2, \cdots, m$. Then the regular representation $\Re$ formed with regard to this basis $A_{\kappa}$ breaks up in the form (44), the degrees $h_{\mu}$ being given by $h_{\mu}=t_{\mu}-t_{\mu-1}$. We say that the $l$-basis $A_{\kappa}$ has been adapted to the chain (45) of $r$-ideals. If we change the $A_{\kappa}$ corresponding to $\mathfrak{S}_{\mu}$, i.e., the $A_{\kappa}$ with $t_{\mu-1}<\kappa \leqq t_{\mu}$, in such a manner that the new basis is still adapted to the chain (45), then $\Re$ undergoes a similarity transformation of the type (3.3C).

2. We assume that the ring $\mathfrak{A}$ contains $n \times K$. Every $r$-ideal $\mathfrak{T}$ is a $K$-left module and a $K$-right module. Then, (11.1A) can be applied. The set $\overline{\mathfrak{T}}=\mathfrak{T} \cap[Z]_{n}$ will be a right ideal of $\overline{\mathfrak{A}}=\mathfrak{A} \cap[Z]_{n}$ considered as an algebra over $Z$. Every right ideal $\overline{\mathfrak{T}}$ of $\overline{\mathfrak{A}}$ will be obtained in this form, if we take $\mathfrak{T}=\mathfrak{M}(\overline{\mathfrak{T}})$.

(12.2A) If $\mathfrak{A}$ is a ring containing $n \times K$, then by

$$
\overline{\mathfrak{T}}=\mathfrak{T} \cap[Z]_{n}, \quad \mathfrak{T}=\mathfrak{M}(\overline{\mathfrak{T}})
$$

there is defined a (1-1) correspondence between the set of the r-ideals $\mathfrak{T}$ of $\mathfrak{A}$ and the set of the r-ideals $\overline{\mathfrak{T}}$ of $\overline{\mathfrak{A}}=\mathfrak{A} \cap[Z]_{n}$. Here $\overline{\mathfrak{A}}$ is considered as an algebra over the centre $Z$ of $K$.

Further, we easily see from (11.1A) that

(12.2B) If an ascending chain of r-ideals of $\mathfrak{A}$ is given, we can choose an $l$-basis $A_{\kappa}$ of $\mathfrak{A}$ which is adapted to this basis such that every $A_{\kappa}$ has coefficients in the centre $Z$.

(12.2C) Let $\bar{A}_{1}, \bar{A}_{2}, \cdots, \bar{A}_{k}$ be an l-basis of $\overline{\mathfrak{A}}$ such that the regular representation $\bar{\Re}$ of $\overline{\mathfrak{A}}$ formed with regard to this basis splits into constituents which are 
irreducible in $Z$. If the same basis $\bar{A}_{\kappa}$ is used for the definition of the regular representation $\Re$ of $\mathfrak{A}$, then $\Re$ splits into constituents which are irreducible in $K$.

3. We now discuss conditions under which the $\mathfrak{S}_{\nu}$ in (44) are completely reducible.

(12.3A) Let $\mathfrak{A}$ be a ring containing $n \times K$ whose radical is $\mathfrak{N}$. Let $(0)=\mathfrak{T}_{0} \subset \mathfrak{T}_{1} \subset \cdots \subset \mathfrak{T}_{m}=\mathfrak{A}$ be a chain of right ideals. In the corresponding splitting (44) of the regular representation $\Re$, the constituent $\mathfrak{S}_{\mu}$ is completely reducible, if and only if $\mathfrak{T}_{\mu} \mathfrak{N} \subseteq \mathfrak{T}_{\mu-1}$.

Proof. If $\mathfrak{I}_{\mu} \mathfrak{N} \subseteq \mathfrak{T}_{\mu-1}$, then $\mathfrak{R}$ will be represented by 0 in $\mathfrak{S}_{\mu}$. From (11.5F) and $(11.4 \mathrm{C})$ it follows that $\mathfrak{S}_{\mu}$ has the radical (0) and hence is completely reducible. Conversely, if $\mathfrak{S}_{\mu}$ is completely reducible, it represents $\mathfrak{N}$ by 0 . Then $\mathfrak{T}_{\mu} \mathfrak{R} \subseteq \mathfrak{T}_{\mu-1}$, as was stated.

The complete reducibility of $\mathfrak{S}_{\mu}$ is, of course, equivalent to the complete reducibility of $\mathfrak{T}_{\mu} / \mathfrak{T}_{\mu-1}$ considered as an additive group with the elements of $K$ as $l$-operators and the elements of $\mathfrak{A}$ as $r$-operators.

In order to obtain the lower Loewy normal form of $\Re$, we must choose $\mathfrak{I}_{m-1}$ as small as possible such that $\mathfrak{I}_{m} \mathfrak{N} \subseteq \mathfrak{T}_{m-1}$; then $\mathfrak{I}_{m-2}$ as small as possible such that $\mathfrak{T}_{m-1} \mathfrak{N} \subseteq \mathfrak{T}_{m-2}$. Thus

(12.3B) Let $\mathfrak{A}$ be a ring containing $n \times K$ whose radical is $\mathfrak{N}$. The lower Loewy normal form of the regular representation $\mathfrak{R}$ of $\mathfrak{A}$ corresponds to the chain of r-ideals (0) $=\mathfrak{N}^{L} \subset \mathfrak{N}^{L-1} \subset \mathfrak{N}^{L-2} \subset \cdots \subset \mathfrak{N} \subset \mathfrak{N}^{0}=\mathfrak{A}\left({ }^{(38}\right)$. The exponent $L$ here is equal to the number $L(\mathfrak{A})=L(\Re)$ of Loewy constituents of $\mathfrak{A}$ and $\Re$ (cf. (8.2B)).

Similarly, we obtain from (12.3A) the theorem that

(12.3C) Under the assumptions of (12.3B), the upper Loewy normal form of $\Re$ corresponds to the series of ideals $(0)=\mathfrak{Q}_{0} \subset \mathfrak{Q}_{1} \subset \cdots \subset \mathfrak{Q}_{L}=\mathfrak{A}$, where $\mathfrak{Q}_{i}$ consists of the l-annihilators of $\mathfrak{R}^{i}$ in $\mathfrak{A}$.

If we consider $\mathfrak{A}$ as an additive group with the elements of $K$ as $l$-operators and the elements of $\mathfrak{A}$ as $r$-operators, we may say that $\mathfrak{N}^{L} \subset \mathfrak{N}^{L-1} \subset \cdots \subset \mathfrak{A}$ and $\mathfrak{Q}_{0} \subset \mathfrak{Q}_{1} \subset \cdots \subset \mathfrak{Q}_{L}$ are the upper and the lower Loewy series of $\mathfrak{A}$.

4. From (12.3B) we see that the degree $\lambda$ of $\mathfrak{R}_{1}(\Re)$ is equal to $k-\nu$ where $k$ is the $l$-rank of $\mathfrak{A}$ and $\nu$ is the $l$-rank of $\Re$. In the notation of (11.4B) $\lambda=\sum k_{\mu}$. But the argument of $\$ 10.1$ easily shows that every $\mathfrak{F}_{\mu}$ appears at least $k_{\mu} / f_{\mu}$ times in $R_{1}(\Re)$. Therefore, the constituents $\mathfrak{F}_{\mu}$ occupy at least $k_{\mu}$ ordinary rows of $\ell_{1}(\Re)$. Because $\lambda=\sum k_{\mu}$, we obtain:

(12.4A) If $\mathfrak{F}_{\mu}$ is an irreducible representation of $\mathfrak{A}$ of l-rank $k_{\mu}$ and of degree $f_{\mu}$, then $\mathfrak{F}_{\mu}$ appears exactly $k_{\mu} / f_{\mu}$ times in the first Loewy constituent $\mathfrak{R}_{1}(\Re)$ of the regular representation $\Re$ of $\mathfrak{A}$.

(38) Cf. Nesbitt [19]. 


\section{BibliogRAPHY}

1. A. A. Albert, Modern Higher Algebra, Chicago, 1937.

2. - Structure of Algebras, American Mathematical Society Colloquium Publications, vol. 24, 1939.

3. R. Brauer, Ueber Systeme hyperkomplexer Zahlen, Mathematische Zeitschrift, vol. 29 (1929), pp. 79-107.

4. R. Brauer and C. Nesbitt, On the regular representation of algebras, Proceedings of the National Academy of Sciences, vol. 23 (1937), pp. 236-240.

5. W. Burnside, Reducibility of any group of linear substitutions, Proceedings of the London Mathematical Society, (2), vol. 3 (1905), pp. 430-434.

6. A. H. Clifford, Representations induced in an invariant subgroup, Annals of Mathematics, (2), vol. 38 (1937), pp. 533-550.

7. M. Deuring, Algebren, Ergebnisse der Mathematik, vol. 4, 1935.

8. H. Fitting, Die Theorie der Automorphismenringe Abelscher Gruppen und ihr Analogon bei nichtkommutativen Gruppen, Mathematische Annalen, vol. 107 (1932), pp. 514-542.

9. G. Frobenius, Theorie der hyperkomplexen Grössen, Sitzungsberichte der Preussischen Akademie der Wissenschaften, 1903, pp. 504-537.

10. G. Frobenius and I. Schur, Ueber die Aequivalenz von Gruppen linearer Substitutionen, Sitzungsberichte der Preussischen Akademie der Wissenschaften, 1906, pp. 209-217.

11. W. Krull, Ueber verallgemeinerte endliche Abelsche Gruppen, Mathematische Zeitschrift, vol. 23 (1925), pp. 161-186.

12. - Theorie und Anwendung der verallgemeinerten Abelschen Gruppen, Sitzungsberichte der Heidelberg Akademie der Wissenschaften, 1926.

13. A. Loewy, Ueber die Reduzibilität der Gruppen linearer homogener Substitutionen, these Transactions, vol. 4 (1903), pp. 44-64.

14. - Ueber die vollständig reduciblen Gruppen, die zu einer Gruppe linearer homogener Substitutionen gehören, these Transactions, vol. 6 (1905), p. 504.

15. — Ueber Matrizen und Differentialkomplexe, I, II, III, Mathematische Annalen, vol. 78 (1917), pp. 1-51, 343-368.

16. C. C. MacDuffee, On the independence of the first and second matrices of an algebra, Bulletin of the American Mathematical Society, vol. 35 (1929), pp. 344-349.

17. F. D. Murnaghan, The Theory of Group Representations, Baltimore, 1928.

18. T. Nakayama, Some studies on regular representations, induced representations and modular representations, Annals of Mathematics, (2), vol. 39 (1938), pp. 361-369.

19. C. Nesbitt, On the regular representations of algebras, Annals of Mathematics, (2), vol. 39 (1938), pp. 634-658.

20. E. Noether, Hyperkomplexe Grössen und Darstellungstheorie, Mathematische Zeitschrift, vol. 30 (1939), pp. 641-692. 541.

21. — Nichtkommutative Algebra, Mathematische Zeitschrift, vol. 37 (1933), pp. 514-

22. O. Ore, Structures and group theory II, Duke Mathematical Journal, vol. 4 (1938), pp. 247-269.

23. L. Pontrjagin, Ueber den algebraischen Inhalt der topologischen Dualitätssätze, Mathematische Annalen, vol. 105 (1931), pp. 165-205.

24. R. Remak, Ueber minimale invariante Untergruppen in der Theorie der endlichen Gruppen, Journal für die reine und angewandte Mathematik, vol. 162 (1930), pp. 1-16.

25. O. Schreier, Ueber den Jordan-Hölderschen Satz, Abhandlungen aus dem mathematischen Seminar der Hamburgischen Universität, vol. 6, pp. 300-302.

26. I. Schur, Neue Begründung der Theorie der Gruppencharaktere, Sitzungsberichte der Preussischen Akademie der Wissenschaften, 1905, pp. 406-432. 
27. - Beiträge zur Theorie der Gruppen linearer Substitutionen, these Transactions, vol. 15 (1909), pp. 159-175.

28. B. L. van der Waerden, Moderne Algebra, 2 vols., Berlin, 1931.

29. - Gruppen von linearen Transformationen, Ergebnisse der Mathematik, vol. 4, 1935.

30. J. L. Wedderburn, Lectures on Matrices, American Mathematical Society Colloquium Publications, vol. 17, 1934.

31. H. Weyl, The Classical Groups, Princeton, 1939.

32. Zassenhaus, Lehrbuch der Gruppentheorie, Leipzig, 1937.

The UnIVERsity OF TORONTO, Toronto, Canada. 